TUM-HEP-615/05

hep-ph/0512234

\title{
Constraining Mass Spectra with Sterile Neutrinos from Neutrinoless Double Beta Decay, Tritium Beta Decay and Cosmology
}

\author{
Srubabati Goswami ${ }^{a, b *}$ and Werner Rodejohann ${ }^{b \dagger}$ \\ ${ }^{a}$ Harish-Chandra Research Institute, Chhatnag Road, \\ Jhunsi, Allahabad 211 019, India \\ ${ }^{b}$ Physik-Department, Technische Universität München, \\ James-Franck-Strasse, D-85748 Garching, Germany
}

\begin{abstract}
We analyze the constraints on neutrino mass spectra with extra sterile neutrinos as implied by the LSND experiment. The various mass related observables in neutrinoless double beta decay, tritium beta decay and cosmology are discussed. Both neutrino oscillation results as well as recent cosmological neutrino mass bounds are taken into account. We find that some of the allowed mass patterns are severely restricted by the current constraints, in particular by the cosmological constraints on the total sum of neutrino masses and by the non-maximality of the solar neutrino mixing angle. Furthermore, we estimate the form of the four neutrino mass matrices and also comment on the situation in scenarios with two additional sterile neutrinos.
\end{abstract}

*email: sruba@ph.tum.de

$\dagger$ 'email: werner_rodejohann@ph.tum.de 


\section{Introduction}

Scenarios with four neutrinos became popular on the wake of the LSND evidence of $\bar{\nu}_{\mu}-\bar{\nu}_{e}$ transitions [1]. Interpreted in terms of neutrino oscillations, the indicated mass scale for LSND is in the $\mathrm{eV}^{2}$ range. Together with the evidence for neutrino oscillations from atmospheric (plus K2K) and solar (plus KamLAND) neutrino observations, requiring mass scales around $10^{-3} \mathrm{eV}^{2}$ and $10^{-4} \mathrm{eV}^{2}$, respectively, a fourth sterile neutrino has to be introduced in order to accommodate the presence of three distinct mass squared differences. A priori, four neutrino scenarios allow for two possible mass patterns:

(i) $2+2$ scenarios, in which two pairs of neutrino states are separated from each other by the LSND mass scale. There are two possibilities for $2+2$ scenarios;

(ii) $3+1$ scenarios, in which one single neutrino state is separated by the LSND mass scale from the other three states. There are four possibilities for $3+1$ scenarios;

Oscillation analyzes in both schemes were performed by a number of authors [2, 3, 4, 5] and also the astrophysical and cosmological implications were investigated [6, 7]. Historically, among the above two alternatives the $3+1$ scenarios were at first relatively disfavored [3] because of the non-observation of oscillations in short baseline experiments like KARMEN [8], Bugey 9] and CDHS [10]. Therefore the 2+2 scenarios were found to be more compatible with the existing data. The sterile neutrino oscillation solution in $2+2$ scenarios was viable for both solar and atmospheric neutrinos. However, SuperKamiokande data disfavored oscillation of the atmospheric $\nu_{\mu}$ to purely sterile neutrinos [1], and later on the SNO data started establishing the neutral current component in the solar $\nu_{e}$ flux [12]. For some time a mixed scenario, where the atmospheric neutrino anomaly is due to $\nu_{\mu}-\nu_{s, \tau}$ and the solar neutrino anomaly is due to $\nu_{e}-\nu_{s, \tau}$, remained compatible with all data [13. However, all recent analyzes show that $2+2$ scenarios are ruled out at a high $\sigma$ from the existing data [14, 15. Both atmospheric and solar neutrino data strongly disfavor oscillations to pure sterile species. This disfavored the $2+2$ scenarios irrespective of whether LSND results are confirmed or not. The most updated analysis in the $3+1$ scheme performed in [14, 15] shows that non-evidence of neutrino oscillation in other short baseline (SBL) experiments combined with atmospheric neutrino data from SuperK and K2K is inconsistent with the LSND signal at 95\% C.L. and only marginal overlaps are found at 99\% C.L. Thus, with increased precision of solar and atmospheric neutrino flux measurements the four neutrino explanation of the LSND anomaly suffered a setback. This led to many alternative explanations of the LSND anomaly including introduction of two sterile neutrinos - the so-called $3+2$ scenario [16] -, CPT violation [17, quantum decoherence effects violating CPT [18, mass varying neutrinos [19, neutrino decay in four neutrino scenarios [20], lepton number violating muon decay [21], decay of a heavy neutrino [22] or extra dimensional aspects [23].

Oscillation experiments can only measure the mass squared differences but not the absolute masses. The most direct and model independent way to measure the absolute masses is via kinematic measurements involving nuclear beta decay. The best bound at present is 
$m_{\beta}<2.3 \mathrm{eV}$ (95\% C.L.) coming from the Mainz tritium beta decay experiment [24]. The KATRIN experiment is expected to increase the sensitivity down to $\sim 0.2 \mathrm{eV}[25]$.

Information on absolute masses can also come from neutrinoless double beta decay $(0 \nu \beta \beta)$. Neutrinoless double beta decay experiments aim at observing the process

$$
(A, Z) \rightarrow(A, Z+2)+2 e^{-} .
$$

This is a lepton number violating process and its observation will establish the Majorana nature of neutrinos [26]. The decay width depends quadratically on the so-called effective mass. We assume here that only the light Majorana neutrinos implied by neutrino oscillation experiments are exchanged in the diagram of $0 \nu \beta \beta$. In the basis in which the charged lepton mass matrix is real and diagonal, the effective mass is then nothing but the absolute value of the ee element of the neutrino mass matrix. The best current limit on the effective mass is given by measurements of ${ }^{76} \mathrm{Ge}$ established by the Heidelberg-Moscow collaboration [27] (with similar results obtained by the IGEX experiment [28])

$$
\langle m\rangle \leq 0.35 \zeta \mathrm{eV}
$$

where $\zeta=\mathcal{O}(1)$ indicates that there is an uncertainty stemming from the nuclear physics involved in calculating the decay width of $0 \nu \beta \beta$. The running projects NEMO3 29] and CUORICINO [30] will be joined in the near future by next generation experiments such as CUORE 31, MAJORANA 32, GERDA [33], EXO 34, MOON [35, COBRA [36], XMASS, DCBA 37, CANDLES [38, CAMEO 39] (for a review see 40]). One can safely expect that values of $\langle m\rangle$ one order of magnitude below the limit from Eq. (11) will be probed within the next, say, 10 years ${ }^{1}$. This means that scales of order $\sqrt{\Delta m_{\text {LSND }}^{2}}$ will be fully probed, and are even under investigation now. Since the effective mass measured in $0 \nu \beta \beta$ also depends on the neutrino mixing angles, the neutrino mass scale and ordering, as well as the mass squared differences, it is possible to obtain additional constraints on sterile neutrino scenarios using neutrinoless double beta decay [42, 43, 44].

Important constraints on sterile neutrinos can also come from cosmology. Inclusion of an extra neutrino, even if sterile, can be in conflict with cosmological observations. The problems are increased if the extra sterile neutrino is massive and has significant mixing with the active species. In particular the Big Bang Nucleosynthesis model of standard cosmology, which explains light element abundances of the Universe, puts constraints on the number of neutrino species. The latest bound found in [45] for instance is $1.7<N_{\nu}<3.0$ at $95 \%$ C.L. and in [46] it is quoted that $N_{\nu}=3.14_{-0.65}^{+0.70}$. The differences in the results are due to different inputs regarding the uncertainties in the primordial He abundance. Observations of the Cosmic Microwave Background and of large scale structures can also constrain the number of neutrino species. A summary of these bounds obtained by various groups including different data sets can be found in [47. The upper limit on the number of neutrinos in these analysis can vary from 6 to 8. A recent bound as quoted in [47] is

\footnotetext{
${ }^{1}$ Not to forget, those experiments aim also to put the controversial [41] evidence of part of the Heidelberg-Moscow collaboration to the test.
} 
$N_{\nu}=4.2_{-1.2}^{+1.7}$ at $95 \%$ C.L. Another important constraint from cosmology comes on the sum of total masses of all the neutrinos, $\Sigma \equiv \sum m_{i}$. For four light neutrinos with degenerate masses the bound is $\Sigma<1.7 \mathrm{eV}$ (95\% C.L.) from WMAP and $2 \mathrm{dF}$ data [4]. For four (five) neutrinos, with one (two) of them carrying a mass, the bound is $\Sigma<1.05$ (1.64) eV (95\% C.L.) [4]. Improvement of these numbers within one order of magnitude is expected [4]. Note that these bounds depend on the priors and data sets used, for slightly more stringent bounds see, e.g., 49. The above constraints can however be evaded if the abundances of sterile neutrinos in the early Universe can be suppressed. This requires going beyond the framework of standard cosmology and introducing mechanisms such as primordial lepton asymmetries [50], low re-heating temperature [51], additional neutrino interactions [52 ${ }^{2}$ etc.

Turning back to oscillations, the MiniBooNE experiment 54 is expected to confirm or refute the LSND signal and is expected to publish results within the next 6 months or so. If MiniBooNE does not confirm the LSND signal, then with the data collected with $10^{21}$ protons on target they can rule out the entire $90 \%$ area allowed by LSND with 4 to $5 \sigma$ [54. If however they confirm the LSND signal then this will give rise to an intriguing situation in what regards the explanation of global oscillation data from accelerator, reactor, atmospheric and solar neutrino experiments. If confirming the LSND result, MiniBooNE can not distinguish the allowed four (or five) neutrino mass spectra. To understand the implied mass and mixing scheme, other observables are therefore crucial. This concerns in particular observables depending on the neutrino masses and ordering. Inasmuch one can use these future measurements to identify the neutrino spectrum is one of the motivations of this work. We stress here that we assume only the neutrino oscillation explanation of the LSND result is correct, i.e., the new physics alternatives (not necessarily predicting a signal for MiniBooNE) put forward in Refs. [17, 18, 19, 20, 21, 22, 23] are not required.

In this paper we examine what constraints from current and future data can be obtained on possible neutrino mass spectra in scenarios with one or more sterile neutrinos. For the four allowed $3+1$ scenarios we give the neutrino masses, their sum as testable in cosmology, the kinematic neutrino mass for tritium experiments and the effective mass in neutrinoless double beta decay. We include the most recent values of mass-squared differences and mixing angles from latest global analyzes of oscillation data. We furthermore reconstruct the possible mass matrices in four neutrino scenarios that are consistent with the current data. Finally, we also comment on $3+2$ scenarios.

The paper is build up as follows: In Section 2 we discuss our parametrization of the four neutrino mixing matrix and summarize the relevant formulae for the neutrino masses, their sum, the kinematic neutrino mass measured in beta decay experiments and the effective mass that can be observed in neutrinoless double beta decay. In Section 3 we apply this framework to $3+1$ scenarios. Approximate forms of the neutrino mass matrices in $3+1$ schemes that are consistent with the current data are given in Section 4. In Section 5 we comment on the above quantities in the $3+2$ scheme, before presenting our summary

\footnotetext{
${ }^{2}$ See however [53].
} 
and conclusions in Section 6. The oscillation probabilities for the relevant short baseline oscillation experiments are delegated to the Appendix. Although $2+2$ scenarios are highly disfavored we also add for the sake of completeness an Appendix on the implications of such scenarios for neutrino masses from cosmology, beta decay and neutrinoless double beta decay. We also discuss the form of mass matrices in the $2+2$ scenarios.

\section{Four Neutrino Mixing and Neutrino Masses}

Neutrino mixing is described by the unitary Pontecorvo-Maki-Nakagawa-Sakata (PMNS) matrix $U$ 55. For four Dirac neutrinos it contains 6 angles $\theta_{12,13,14,23,24,34}$ and three phases $\delta_{13,14,24}$ ("Dirac phases"). Their Majorana nature, which we shall assume, adds another three phases ("Majorana phases"), which do not have any consequences in neutrino oscillations [56]. We parametrize $U$ as

$$
U=R_{34} \tilde{R}_{24} \tilde{R}_{14} R_{23} \tilde{R}_{13} R_{12} P=\left(\begin{array}{cccc}
U_{e 1} & U_{e 2} & U_{e 3} & U_{e 4} \\
U_{\mu 1} & U_{\mu 2} & U_{\mu 3} & U_{\mu 4} \\
U_{\tau 1} & U_{\tau 2} & U_{\tau 3} & U_{\tau 4} \\
U_{s 1} & U_{s 2} & U_{s 3} & U_{s 4}
\end{array}\right)
$$

where the $R_{i j}$ represent rotations in $i j$ generation space, for instance:

$$
R_{34}=\left(\begin{array}{cccc}
1 & 0 & 0 & 0 \\
0 & 1 & 0 & 0 \\
0 & 0 & c_{34} & s_{34} \\
0 & 0 & -s_{34} & c_{34}
\end{array}\right) \quad \text { or } \quad \tilde{R}_{14}=\left(\begin{array}{cccc}
c_{14} & 0 & 0 & s_{14} e^{-i \delta_{14}} \\
0 & 1 & 0 & 0 \\
0 & 0 & 1 & 0 \\
-s_{14} e^{i \delta_{14}} & 0 & 0 & c_{14}
\end{array}\right)
$$

with the usual notation $s_{i j}=\sin \theta_{i j}$ and $c_{i j}=\cos \theta_{i j}$. The diagonal matrix $P$ contains the three Majorana phases, which we denote $\alpha, \beta$ and $\gamma$ :

$$
P=\operatorname{diag}\left(1, e^{-i \alpha / 2}, e^{-i\left(\beta / 2-\delta_{13}\right)}, e^{-i\left(\gamma / 2-\delta_{14}\right)}\right) .
$$

For most purposes it is sufficient to analyze the individual experimental data in a two-flavor framework. Depending on the neutrino mass spectrum, one can then identify certain elements of the PMNS matrix with the mixing angle in a two-neutrino oscillation probability. For the parameters governing solar (and KamLAND), atmospheric (and K2K) and short baseline reactor neutrino oscillation it holds at $3 \sigma$ [57, 58]

$$
\begin{gathered}
7.0 \times 10^{-5} \mathrm{eV}^{2}<\Delta m_{\odot}^{2}<9.3 \times 10^{-5} \mathrm{eV}^{2} \\
0.25<\sin ^{2} \theta_{\odot}<0.40
\end{gathered}
$$

with best-fit values of $\Delta m_{\odot}^{2}=8 \times 10^{-5} \mathrm{eV}^{2}$ and $\sin ^{2} \theta_{\odot}=0.31$. The atmospheric mass squared difference and mixing angle at $3 \sigma$ are known within [15]

$$
\begin{gathered}
1.3 \times 10^{-3} \mathrm{eV}^{2}<\Delta m_{\mathrm{A}}^{2}<4.2 \times 10^{-3} \mathrm{eV}^{2}, \\
0.33<\sin ^{2} \theta_{\mathrm{A}}<0.66
\end{gathered}
$$



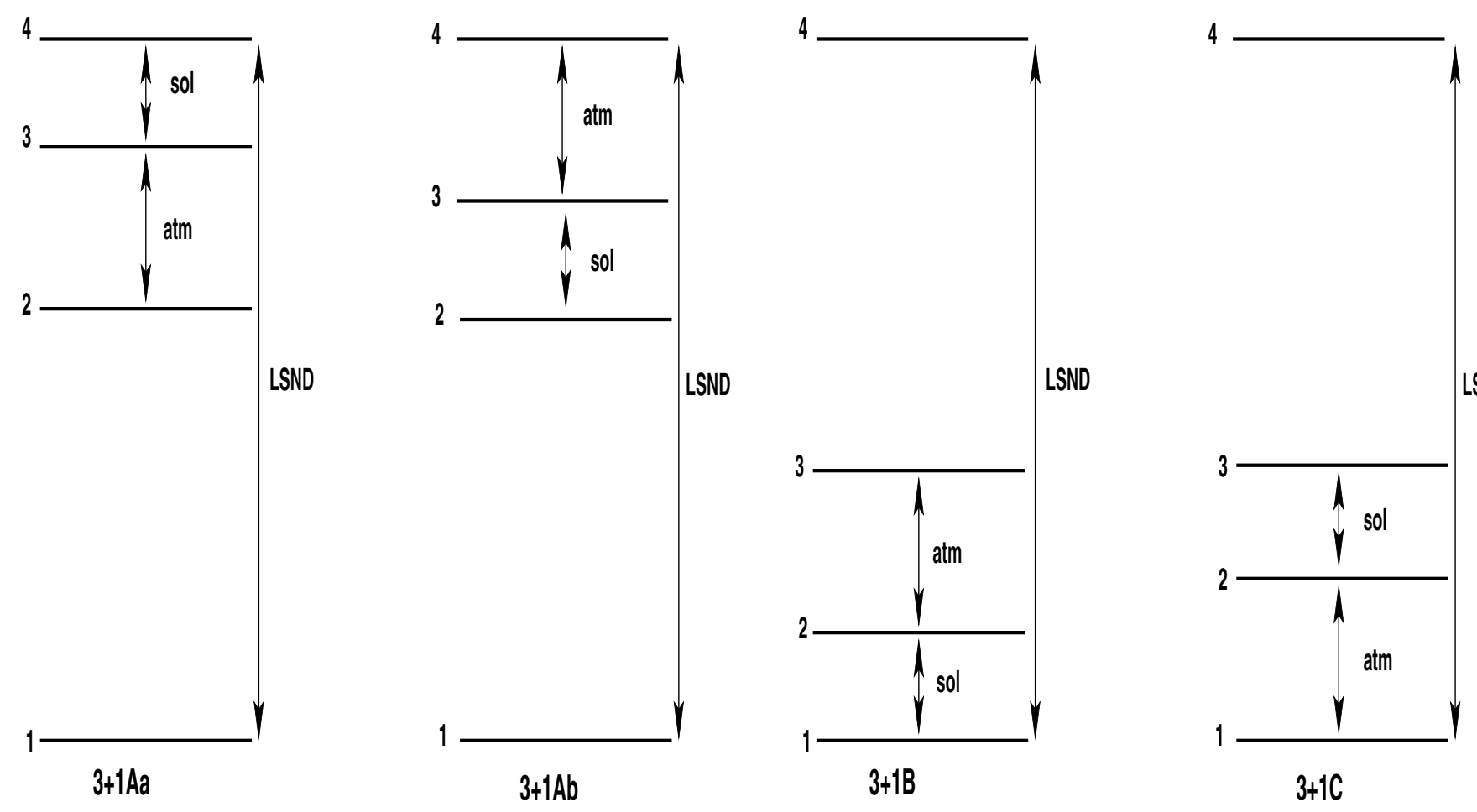

Figure 1: The four allowed 3+1 mass orderings.

with best-fit values of $\Delta m_{\mathrm{A}}^{2}=2.2 \times 10^{-3} \mathrm{eV}^{2}$ and $\sin ^{2} \theta_{\mathrm{A}}=1 / 2$. The mixing angle $\theta_{13}$ at $3 \sigma$ is restricted to lie below the value [59]

$$
\sin ^{2} \theta_{\mathrm{CHOOZ}}<0.044
$$

In Appendix $\mathrm{A}$ we give the expressions for the oscillation probabilities in short baseline accelerator and reactor experiments and also for the $1 \mathrm{~km}$ reactor experiment CHOOZ. For all the short baseline experiments excepting CHOOZ the one mass scale dominance approximation holds to a good precision. A comparison of the probabilities in the $3+1$ picture (recall that $2+2$ scenarios are highly disfavored) with the two generation LSND probability reveals that $\sin ^{2} 2 \theta_{\text {LSND }}$ in $3+1$ scenarios is always of the form $4\left|U_{e i}\right|^{2}\left|U_{\mu i}\right|^{2}$, where $\left|U_{e i}\right|^{2}$ is constrained to be small from Bugey reactor and solar neutrino data and $\left|U_{\mu i}\right|^{2}$ is constrained to be small from CDHS and atmospheric data. The index $i$ depends on the mass ordering. In Ref. [14, 15] the allowed area in the $3+1$ schemes is given in the $\Delta m_{\mathrm{LSND}}^{2}-\sin ^{2} 2 \theta_{\mathrm{LSND}}$ plane. The plot shows two overlap points at $99 \%$ C.L. corresponding to

$$
\left(\Delta m_{\mathrm{LSND}}^{2}, \sin ^{2} 2 \theta_{\mathrm{LSND}}\right)=\left(0.9 \mathrm{eV}^{2}, 0.002\right) \text { and }\left(1.8 \mathrm{eV}^{2}, 0.001\right)
$$




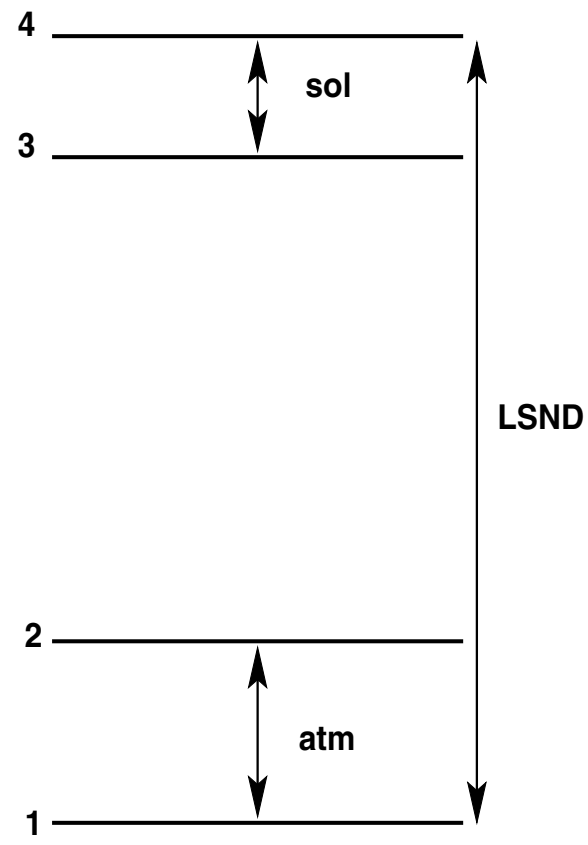

$2+2 A$
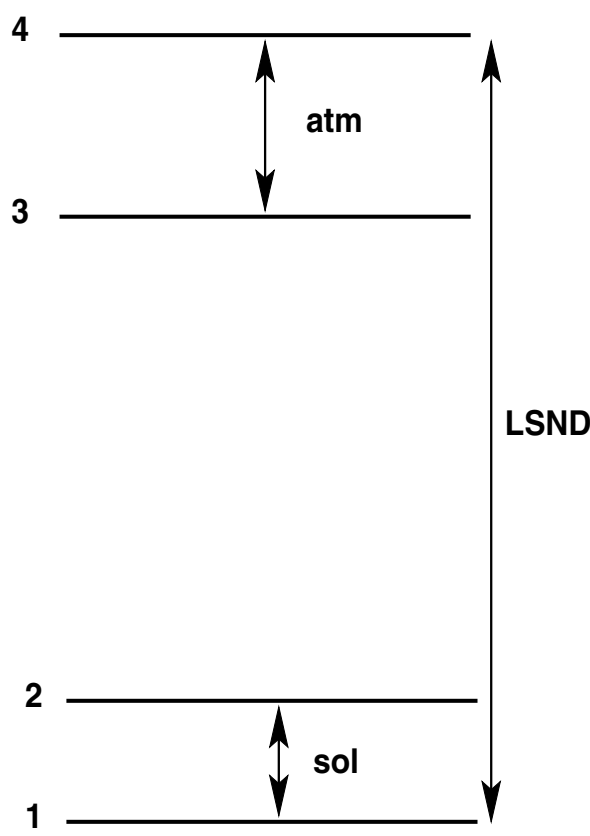

2+2B

Figure 2: The two allowed 2+2 mass orderings.

In our following analysis we take these two allowed values as the illustrative values of $\Delta m_{\mathrm{LSND}}^{2}$ and take $\left|U_{e i}\right|^{2}$ as 0.008 and 0.004 , respectively ${ }^{3}$. It is to be noted that the MiniBooNE sensitivity plots in, e.g., Ref. [73], give an allowed region around $\Delta m_{\mathrm{LSND}}^{2}=0.9$ $\mathrm{eV}^{2}$, which would be obtained if they confirm the LSND signal [73]. Another allowed region is found for which

$$
\Delta m_{\mathrm{LSND}}^{2}=(0.2-0.5) \mathrm{eV}^{2} \text { and } \sin ^{2} 2 \theta_{\mathrm{LSND}}=0.01-0.04 .
$$

We therefore give in the following another set of plots for which we allow $\Delta m_{\mathrm{LSND}}^{2}$ and $\sin ^{2} 2 \theta_{\text {LSND }}$ to vary in this range. To extract $\left|U_{e i}\right|^{2}$ from $\sin ^{2} 2 \theta_{\text {LSND }}$ we assume that $\left|U_{e i}\right| \simeq\left|U_{\mu i}\right|$. With this approximation we have $4\left|U_{e i}\right|^{4}=\sin ^{2} 2 \theta_{\mathrm{LSND}}$, which gives $\left|U_{e i}\right|^{2} \simeq$ $0.05-0.1$. It is to be noted that this range is not allowed at $99 \%$ C.L. according to the analysis of [14. Nevertheless we take this range as an illustrative example to compare with the other two cases with a relatively higher $\Delta m_{\mathrm{LSND}}^{2}$ and lower $\sin ^{2} 2 \theta_{\mathrm{LSND}}$.

The neutrino mass matrix is given by

$$
m_{\nu}=U^{*} m_{\nu}^{\text {diag }} U^{\dagger}, \text { where } m_{\nu}^{\text {diag }}=\operatorname{diag}\left(m_{1}, m_{2}, m_{3}, m_{4}\right) .
$$

We will order the four neutrinos such that $m_{4}>m_{3}>m_{2}>m_{1}$ so that $\Delta m_{\mathrm{LSND}}^{2}$ is always given by $\Delta m_{\mathrm{LSND}}^{2}=m_{4}^{2}-m_{1}^{4} \equiv \Delta m_{41}^{2}$. Depending on the relative ordering within the

${ }^{3}$ This corresponds to $\left|U_{\mu i}\right|^{2}=0.065$, which is the highest allowed value of $\left|U_{\mu i}\right|^{2}$ at $99 \%$ C.L. according to [14. 

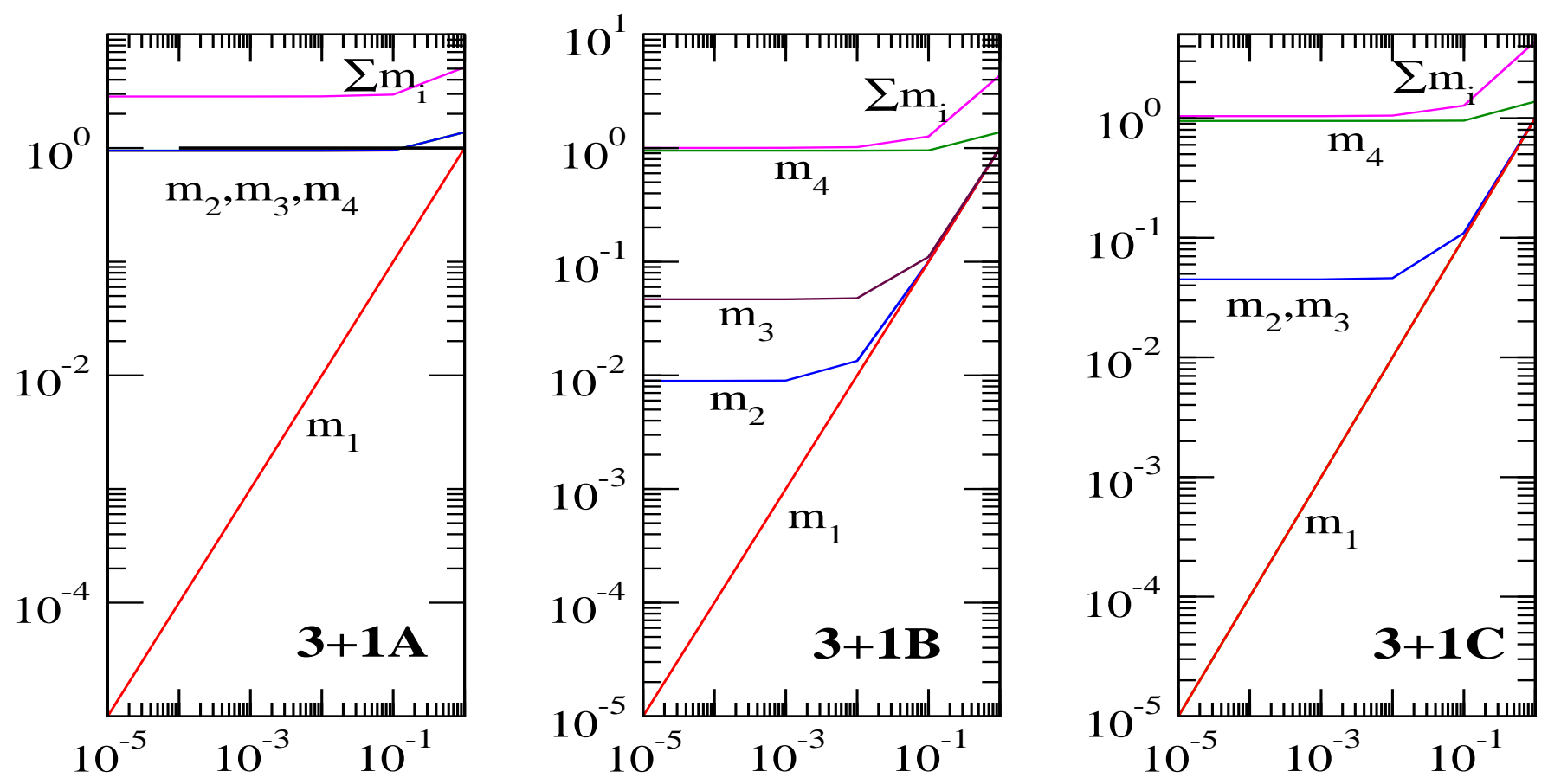

Figure 3: The individual neutrino masses $m_{1,2,3,4}$ and their sum $\Sigma$ as a function of the smallest neutrino mass $m_{1}$. We have $3+1 \mathrm{Aa}$ and $3+1 \mathrm{Ab}$ on the left (they generate basically identical results), $3+1 \mathrm{~B}$ in the middle and $3+1 \mathrm{C}$ on the right. We chose $\Delta m_{\mathrm{LSND}}^{2}=0.9$ $\mathrm{eV}^{2}$ and fixed $\Delta m_{\odot}^{2}$ and $\Delta m_{\mathrm{A}}^{2}$ to their best-fit values.

scheme, there are four possibilities for $3+1$ and two possibilities for $2+2$. We display all six cases in Figs. 1 and 2, One might compare this situation with the three-flavor case, where there are only two possibilities, the normal and inverted ordering. The other two mass differences correspond to $\Delta m_{\odot}^{2}$ and $\Delta m_{\mathrm{A}}^{2}$. One can determine the individual masses as functions of the smallest mass $m_{1}$ and the three mass squared differences:

$$
\begin{gathered}
m_{2}=\sqrt{m_{1}^{2}+\Delta m_{21}^{2}}, \\
m_{3}=\sqrt{m_{1}^{2}+\Delta m_{21}^{2}+\Delta m_{32}^{2}}, \\
m_{4}=\sqrt{m_{1}^{2}+\Delta m_{21}^{2}+\Delta m_{32}^{2}+\Delta m_{43}^{2}}=\sqrt{m_{1}^{2}+\Delta m_{\mathrm{LSND}}^{2}} .
\end{gathered}
$$

As an immediate application, we can then calculate the sum of neutrino masses $\Sigma$,

$$
\Sigma=m_{1}+m_{2}+m_{3}+m_{4}
$$

for which interesting constraints from cosmology apply. Since the individual neutrino masses $m_{1,2,3,4}$ depend crucially on the mass spectrum, their sum $\Sigma$ will do so as well. For the four possible $3+1$ neutrino spectra from Fig. 1 we display the neutrino masses and their sum $\Sigma$ as a function of the smallest mass in Fig. 3 . 
When in addition the mixing matrix elements of the PMNS matrix are specified, one can determine $m_{\beta}$, the parameter measured in the direct neutrino mass searches in nuclear beta decay experiments such as KATRIN. We will denote this parameter the "kinematic mass". It is given by:

$$
m_{\beta}=\sqrt{\left|U_{e 1}\right|^{2} m_{1}^{2}+\left|U_{e 2}\right|^{2} m_{2}^{2}+\left|U_{e 3}\right|^{2} m_{3}^{2}+\left|U_{e 4}\right|^{2} m_{4}^{2}} .
$$

With the inclusion of mixing, the parameters entering the mass measured in beta decay can be expressed in terms of the lowest mass, the mixing matrix elements $\left|U_{e i}\right|^{2}$ and the mass squared differences. Consequently this quantity can also put constraints on the possible mass schemes and their ordering [60. For three neutrino frameworks this has no observable effect since the future sensitivity on $m_{\beta}$ corresponds to quasi-degenerate neutrinos, for which unitarity of the PMNS matrix leads to no dependence on the mixing matrix elements and for which the normal and inverted ordering generate identical results. In the various four neutrino scenarios to be discussed in the following, this will change.

Neutrinoless double beta decay experiments are sensitive to the effective mass which is given as

$$
\begin{gathered}
\langle m\rangle=\left|\sum U_{e i}^{2} m_{i}\right|=\left.|| U_{e 1}\right|^{2} m_{1}+\left|U_{e 2}\right|^{2} m_{2} e^{i \alpha}+\left|U_{e 3}\right|^{2} m_{3} e^{i \beta}+\left|U_{e 4}\right|^{2} m_{3} e^{i \gamma} \mid \\
=\left|m_{1} c_{12}^{2} c_{13}^{2} c_{14}^{2}+m_{2} e^{i \alpha} c_{13}^{2} c_{14}^{2} s_{12}^{2}+m_{3} e^{i \beta} c_{14}^{2} s_{13}^{2}+m_{4} e^{i \gamma} s_{14}^{2}\right| \\
=c_{14}^{2}\left|c_{13}^{2}\left(m_{1} c_{12}^{2}+m_{2} e^{i \alpha} s_{12}^{2}+m_{3} e^{i \beta} t_{13}^{2}\right)+m_{4} e^{i \gamma} t_{14}^{2}\right| .
\end{gathered}
$$

We defined here $t_{i j}=\tan \theta_{i j}$. As can be seen, $\langle m\rangle$ is sensitive to the Majorana phases which may be present in the neutrino mass matrix. The three Dirac phases do not appear in $\langle m\rangle$. The effective mass depends on 10 out of the 16 parameters of the general $4 \times 4$ neutrino mass matrix. This might be compared with the three-flavor case, in which $\langle m\rangle$ depends on 7 out of a total of 9 parameters. Moreover, as in the three-flavor case (for recent analyzes, see [61, 62]), there is a strong dependence on the mass spectrum.

We conclude that the three mass related observables $\langle m\rangle, m_{\beta}$ and $\Sigma$ are powerful tools to discriminate among the various possible mass orderings. This will be the subject of the next Section.

\section{Neutrino Masses and Neutrinoless Double Beta De- cay in $3+1$ Scenarios}

In the next Subsections we discuss the predictions for the sum of neutrino masses, the neutrino mass measured in nuclear beta decay experiments and the effective mass measured in neutrinoless double beta decay in the different $3+1$ scenarios. A common feature of the effective mass is that it can be expressed as a known three-flavor contribution obtained, e.g., in 61, 62] plus an additional term related to the LSND scale. 


\subsection{Neutrino Masses and Neutrinoless Double Beta Decay in Scenarios 3+1Aa and $3+1 \mathrm{Ab}$}

As can be seen in Fig. 1, the scenarios 3+1Aa and 3+1Ab have three quasi-degenerate neutrinos with a mass given by the LSND scale and a fourth, lightest state separated by the LSND scale. It holds that $\Delta m_{\odot}^{2}=m_{4}^{2}-m_{3}^{2}, \Delta m_{\mathrm{A}}^{2}=m_{3}^{2}-m_{2}^{2}$ and $\Delta m_{\odot}^{2}=m_{3}^{2}-m_{2}^{2}$, $\Delta m_{\mathrm{A}}^{2}=m_{4}^{2}-m_{3}^{2}$, respectively. For the $3+1$ Aa case we can use Eq. (13) to express the masses in terms of the smallest mass and the three mass squared differences as

$$
\begin{gathered}
m_{2}=\sqrt{m_{1}^{2}+\Delta m_{\mathrm{LSND}}^{2}-\Delta m_{\odot}^{2}-\Delta m_{\mathrm{A}}^{2}}, \\
m_{3}=\sqrt{m_{1}^{2}+\Delta m_{\mathrm{LSND}}^{2}-\Delta m_{\odot}^{2}}, \\
m_{4}=\sqrt{m_{1}^{2}+\Delta m_{\mathrm{LSND}}^{2}} .
\end{gathered}
$$

For the $3+1 \mathrm{Ab}$ case $\Delta m_{\mathrm{A}}^{2}$ and $\Delta m_{\odot}^{2}$ replace each other. Since the neutrino masses are governed mainly by $\Delta m_{\mathrm{LSND}}^{2}$, the predictions of scenarios $3+1 \mathrm{Aa}$ and $3+1 \mathrm{Ab}$ for all mass related observables are almost identical [44] and therefore we treat these two cases together. Since the minimal mass of the three quasi-degenerate neutrinos is $\sqrt{\Delta m_{\mathrm{LSND}}^{2}}$, it follows for the sum of neutrino masses that

$$
\Sigma^{3+1 \mathrm{Aa}, \mathrm{b}} \gtrsim 3 \sqrt{\Delta m_{\mathrm{LSND}}^{2}} .
$$

The left panel of Figure 3 shows the four masses and their sum $\Sigma$ as a function of the smallest mass $m_{1}$. The three heavier masses are indistinguishable and much larger than the lightest state unless $m_{1} \simeq 1 \mathrm{eV}$. In Figure 3] we have fixed $\Delta m_{\mathrm{LSND}}^{2}$ at $0.9 \mathrm{eV}^{2}$ and hence $\Sigma^{3+1 \mathrm{Aa}, \mathrm{b}} \gtrsim 2.8 \mathrm{eV}$. It follows that these two schemes would already be in conflict with a cosmological limit of $\Sigma=1 \mathrm{eV}$ unless $\Delta m_{\mathrm{LSND}}^{2} \lesssim 0.1 \mathrm{eV}^{2}$. Nevertheless, let us discuss these scenarios further. One would expect that $m_{1} \ll m_{2,3,4}$ holds, so that the fourth state with mass $m_{1}$ effectively decouples in what regards the predictions.

Neutrino data implies in scenario $3+1 \mathrm{Aa}(3+1 \mathrm{Ab})$ that $\sin ^{2} 2 \theta_{\mathrm{LSND}}=4\left|U_{e 1}\right|^{2}\left|U_{\mu 1}\right|^{2}$ (see Appendix $\mathrm{A}$ ) and that $\sin ^{2} \theta_{\mathrm{CHOOZ}} \simeq\left|U_{e 2}\right|^{2}\left(\sin ^{2} \theta_{\mathrm{CHOOZ}} \simeq\left|U_{e 4}\right|^{2}\right)$. One furthermore has $\left|U_{e 3}\right|^{2} \simeq \cos ^{2} \theta_{\odot}$ and $\left|U_{e 4}\right|^{2} \simeq \sin ^{2} \theta_{\odot}(\operatorname{case} 3+1 \mathrm{Aa})$ or $\left|U_{e 3}\right|^{2} \simeq \cos ^{2} \theta_{\odot}$ and $\left|U_{e 2}\right|^{2} \simeq \sin ^{2} \theta_{\odot}$ (case $3+1 \mathrm{Ab})$.

To an excellent approximation, the predictions for neutrinoless double beta decay are very similar to the three-flavor case with quasi-degenerate neutrinos 44. In that case the normal and inverted mass ordering can not be distinguished via $0 \nu \beta \beta$, since their predictions differ only by corrections of order $\Delta m_{\mathrm{A}}^{2} / m_{0}$, where $m_{0}$ is the common three-neutrino mass scale. Analogously, the scenarios $3+1 \mathrm{Aa}$ and $3+1 \mathrm{Ab}$ can not be distinguished via $0 \nu \beta \beta$, since they generate identical results up to corrections of order $\Delta m_{\mathrm{A}}^{2} / \Delta m_{\mathrm{LSND}}^{2}$. For $3+1 \mathrm{Aa}$, one has

$$
\begin{gathered}
\left.\langle m\rangle^{3+1 \mathrm{Aa}} \simeq\left|\sqrt{\Delta m_{\mathrm{LSND}}^{2}+m_{1}^{2}}\left(c_{\odot}^{2}+s_{\odot}^{2} e^{i(\alpha-\beta)}+\sin ^{2} \theta_{\mathrm{CHOOZ}} e^{i(\gamma-\beta)}\right)+e^{-i \beta} m_{1}\right| U_{e 1}\right|^{2} \mid \\
\left.\equiv\left|\langle m\rangle_{3}^{\mathrm{QD}}+m_{1} e^{-i \beta}\right| U_{e 1}\right|^{2} \mid .
\end{gathered}
$$




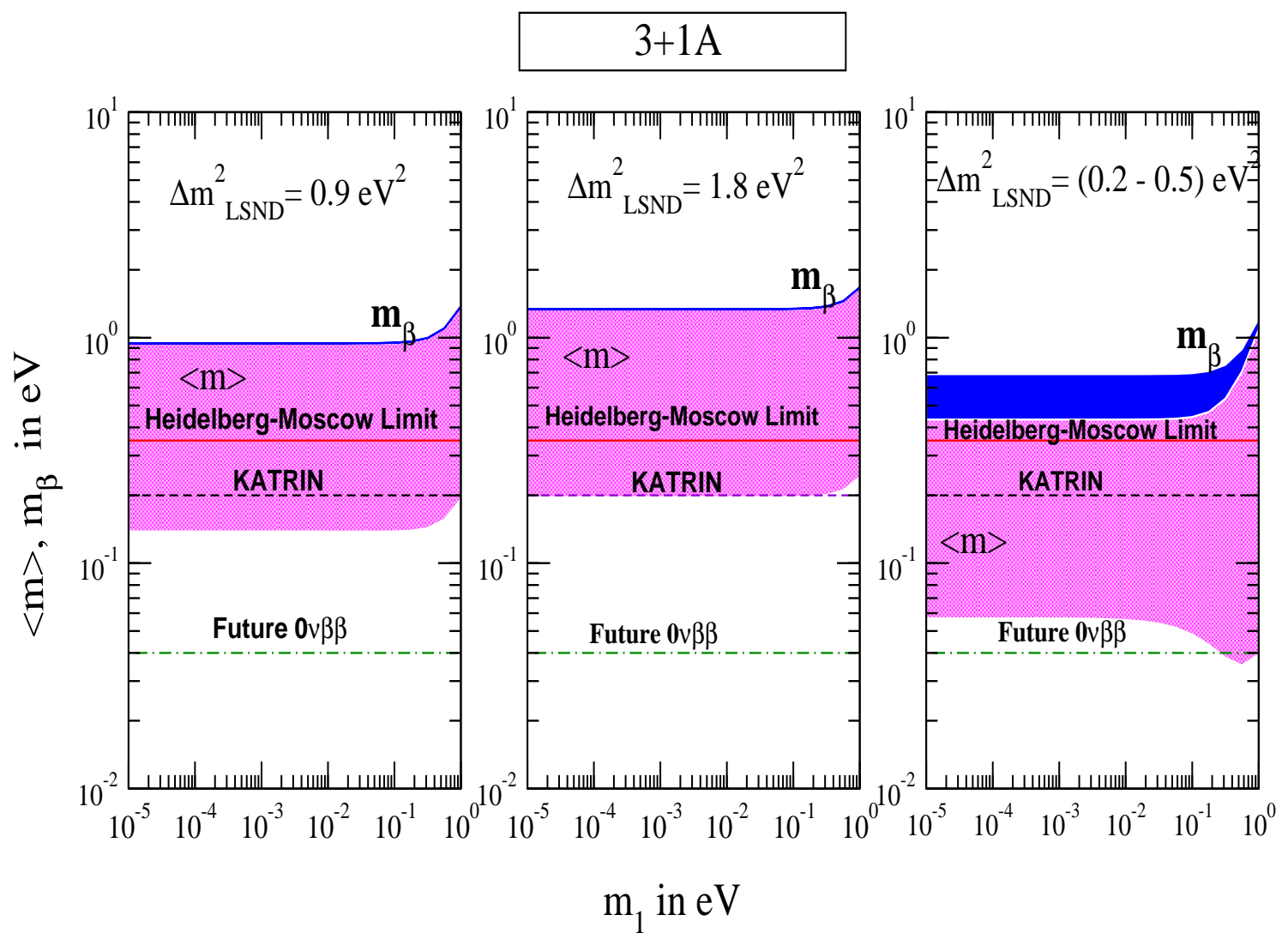

Figure 4: The effective mass as a function of the lowest mass in scenario $3+1 \mathrm{~A}$. The left most column is for $\Delta m_{\mathrm{LSND}}^{2}=0.9 \mathrm{eV}^{2}$ and the middle column is for $\Delta m_{\mathrm{LSND}}^{2}=1.8 \mathrm{eV}^{2}$. The right most column is for $\Delta m_{\mathrm{LSND}}^{2}$ varied between $(0.2-0.5) \mathrm{eV}^{2}$. The other parameters are varied in their current $3 \sigma$ allowed range and all the phases are varied between 0 and $2 \pi$. Also shown is the mass $m_{\beta}$ that will be measured in beta decay experiments, the current and a prospective future limit on the effective mass and the future KATRIN limit.

We have defined $c_{\odot}=\cos \theta_{\odot}, s_{\odot}=\sin \theta_{\odot}$ and used that $\Delta m_{\mathrm{LSND}}^{2} \gg \Delta m_{\mathrm{A}}^{2} \gg \Delta m_{\odot}^{2}$. We also have defined

$$
\begin{gathered}
\langle m\rangle_{3}^{\mathrm{QD}} \equiv m_{0}\left(\cos ^{2} \theta_{\odot}+e^{i(\alpha-\beta)} \sin ^{2} \theta_{\odot}+e^{i(\gamma-\beta)} \sin ^{2} \theta_{\mathrm{CHOOZ}}\right) \\
\text { with }\left|\langle m\rangle_{3}^{\mathrm{QD}}\right| \lesssim m_{0} \frac{1-\tan ^{2} \theta_{12}-2 \sin ^{2} \theta_{\mathrm{CHOOZ}}}{1+\tan ^{2} \theta_{\odot}} \simeq m_{0} \cos 2 \theta_{\odot},
\end{gathered}
$$

with $m_{0}=\sqrt{\Delta m_{\mathrm{LSND}}^{2}+m_{1}^{2}}$. The quantity $\langle m\rangle_{3}^{\mathrm{QD}}$ is just the usual three-flavor effective mass with the common mass scale given by the LSND scale. The contribution of $\langle m\rangle_{3}^{\mathrm{QD}}$ is at least one order of magnitude above the term corresponding to the LSND scale: for instance, if $m_{1}=0.3 \mathrm{eV}, \Delta m_{\mathrm{LSND}}^{2}=0.9 \mathrm{eV}^{2}$, and with $\cos 2 \theta_{\odot} \simeq 0.2-0.5$, one has $\langle m\rangle_{3}^{\mathrm{QD}} \gtrsim 0.2 \mathrm{eV}$ and $m_{1} \sin ^{2} 2 \theta_{\mathrm{LSND}} \simeq 0.002 \mathrm{eV}$. 
Hence, neglecting further $m_{1}^{2}$ with respect to $\Delta m_{\mathrm{LSND}}^{2}$, and also $\sin ^{2} \theta_{\mathrm{CHOOZ}}$, one finds

$$
\langle m\rangle^{3+1 \mathrm{Aa}} \simeq \sqrt{\Delta m_{\mathrm{LSND}}^{2}} \sqrt{1-\sin ^{2} 2 \theta_{\odot} \sin ^{2}(\beta-\gamma) / 2} .
$$

Case $3+1 \mathrm{Ab}$ is obtained by replacing $\alpha$ with $\gamma$. Due to the non-maximal solar neutrino mixing, the effective mass can not vanish in case of scenarios $3+1 \mathrm{Aa}$ and $3+1 \mathrm{Ab}$, its range is given by $\sqrt{\Delta m_{\mathrm{LSND}}^{2}} \cos 2 \theta_{\odot} \lesssim\langle m\rangle^{3+1 \mathrm{Aa}, \mathrm{b}} \lesssim \sqrt{\Delta m_{\mathrm{LSND}}^{2}}$. Therefore, for $\Delta m_{\mathrm{LSND}}^{2}=0.9$ $\mathrm{eV}^{2}$ it follows that $\langle m\rangle_{\min } \simeq 0.19 \mathrm{eV}$ whereas for $\Delta m_{\mathrm{LSND}}^{2}=1.8 \mathrm{eV}^{2}$ we have $\langle m\rangle_{\min } \simeq 0.27$ $\mathrm{eV}$. If $\Delta m_{\mathrm{LSND}}^{2}$ is in the range $(0.2-0.5) \mathrm{eV}^{2}$, then $\langle m\rangle_{\min }$ will be somewhat lower. This approximate behavior is reproduced in Figure 4 where we have plotted the effective mass as a function of the smallest mass. Also included in the Figure is the predicted value of the parameter $m_{\beta}$, together with the anticipated KATRIN limit on $m_{\beta}$ of $0.2 \mathrm{eV}$. For comparison, we also gave the present Heidelberg-Moscow bound on the effective mass, together with a prospective future limit of $0.04 \mathrm{eV}$. Note that the minimum values of $\langle m\rangle$ in the limit of vanishingly small $m_{1}$ are slightly lower than those mentioned above because of the non-zero value of $\sin ^{2} \theta_{\mathrm{CHOOZ}}$. The limit on the effective mass of $0.35 \zeta \mathrm{eV}$ rules out part of the predicted range: for instance, if $\sin ^{2} \theta_{\odot}=0.28$ and $\Delta m_{\mathrm{LSND}}^{2}=0.9 \mathrm{eV}^{2}$, then $\sin ^{2}(\beta-\gamma) / 2 \gtrsim 0.93$ to obey the constraint of $\langle m\rangle \lesssim 0.35 \mathrm{eV}$. For $\sin ^{2} \theta_{\odot}=0.31(0.25 ; 0.40)$ and $\Delta m_{\mathrm{LSND}}^{2}=0.9 \mathrm{eV}^{2}$, we have that $\langle m\rangle \gtrsim 0.36(0.48 ; 0.19) \mathrm{eV}$, whereas for $\Delta m_{\mathrm{LSND}}^{2}=1.8$ $\mathrm{eV}^{2}$ it holds $\langle m\rangle \gtrsim 0.51(0.69 ; 0.27) \mathrm{eV}$. Therefore, if $\sin ^{2} \theta_{\odot}$ turns out to be on the lower side of its currently allowed range, then scenarios $3+1 \mathrm{Aa}$, b face serious problems with the constraints from $0 \nu \beta \beta$.

The kinematic neutrino mass in scenarios $3+1 \mathrm{Aa}, \mathrm{b}$ is directly given by the LSND scale:

$$
m_{\beta}^{3+1 \mathrm{Aa}, \mathrm{b}} \simeq \sqrt{\Delta m_{\mathrm{LSND}}^{2}} .
$$

For fixed $\Delta m_{\mathrm{LSND}}^{2}$ the prediction for $m_{\beta}$ is therefore a line, whereas when a range of values for $\Delta m_{\mathrm{LSND}}^{2}$ is given, we also have a range of values for $m_{\beta}$.

We can summarize the situation ${ }^{4}$ for scenarios $3+1 \mathrm{Aa}, \mathrm{b}$ as follows:

$$
\langle m\rangle^{3+1 \mathrm{Aa}, \mathrm{b}} \simeq m_{\beta}^{3+1 \mathrm{Aa}, \mathrm{b}} \sqrt{1-\sin ^{2} 2 \theta_{\odot} \sin ^{2} \phi} \simeq \frac{\Sigma^{3+1 \mathrm{Aa}, \mathrm{b}}}{3} \sqrt{1-\sin ^{2} 2 \theta_{\odot} \sin ^{2} \phi},
$$

where $\phi$ is some combination of Majorana phases. Some constraints on these parameters might be obtained in this scenario. The kinematic mass $m_{\beta}$ predicted by this scenario is much above the KATRIN sensitivity and it will be disfavored if KATRIN confirms $m_{\beta}$ around $0.2 \mathrm{eV}$. The same is true when cosmological searches do not find a signal close to their current bounds. Future limits on the effective mass below roughly $0.05 \mathrm{eV}$ will also rule out scenarios $3+1 \mathrm{Aa}, \mathrm{b}$. A limit of $0.1 \mathrm{eV}$ will rule out the two overlap points at 0.9 and $1.8 \mathrm{eV}^{2}$.

\footnotetext{
${ }^{4}$ We note here that the predictions for $0 \nu \beta \beta$ and $m_{\beta}$ are identical (i.e., up to corrections of order $\Delta m_{\mathrm{A}}^{2} / \Delta m_{\mathrm{LSND}}^{2}$ ) to the one of (the highly disfavored) scenario $2+2 \mathrm{~A}$, treated in Appendix $\mathrm{B}$ Therefore, one can not distinguish these scenarios via $0 \nu \beta \beta$ or tritium decay experiments. However, since one has $\Sigma^{2+2 \mathrm{~A}} \simeq \frac{2}{3} \Sigma^{3+1 \mathrm{Aa}, \mathrm{b}}$, there would be a chance to distinguish them via cosmological measurements.
} 


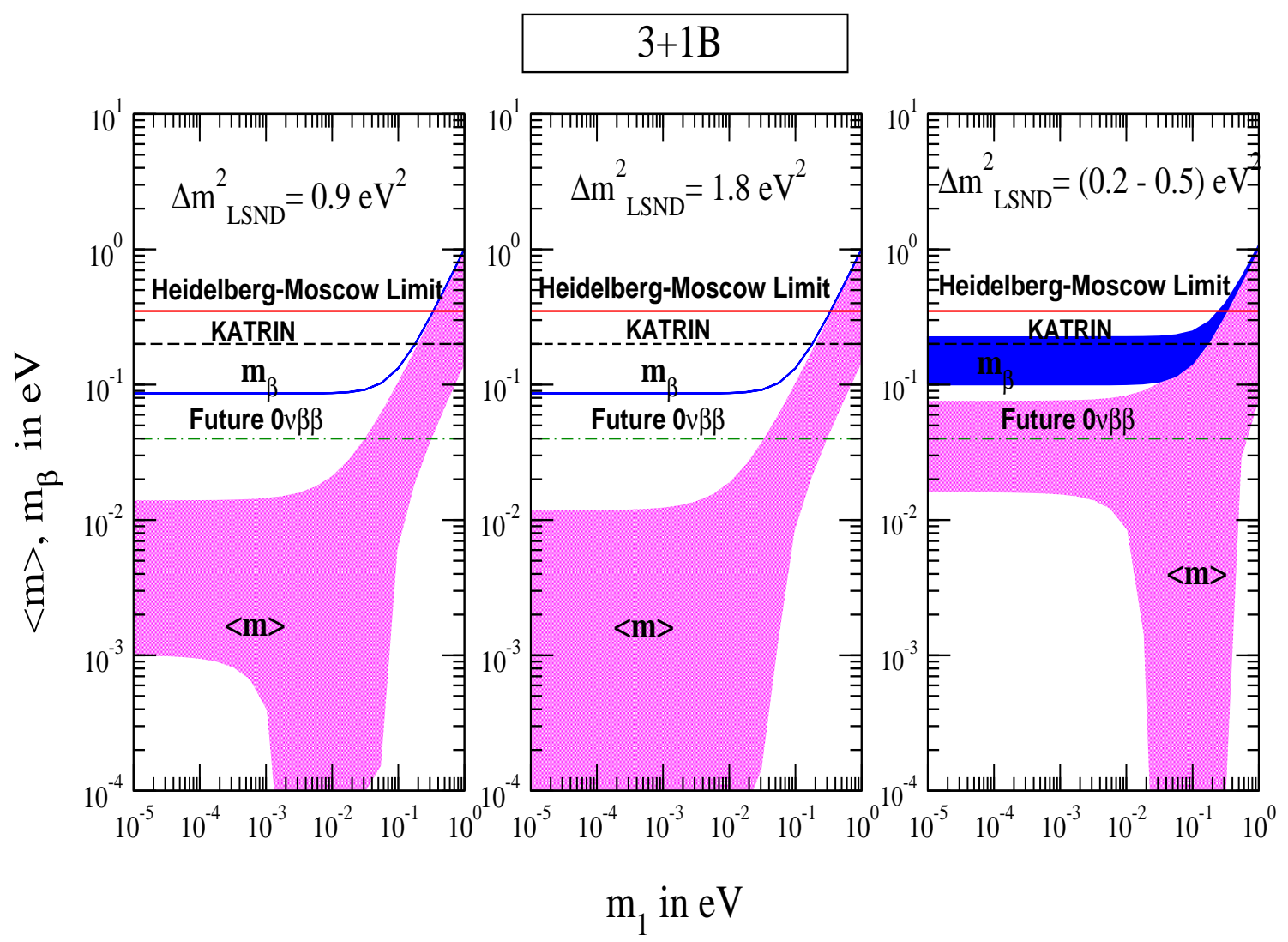

Figure 5: Same as previous Figure for scenario 3+1B.

\subsection{Neutrino Masses and Neutrinoless Double Beta Decay in Scenario $3+1 B$}

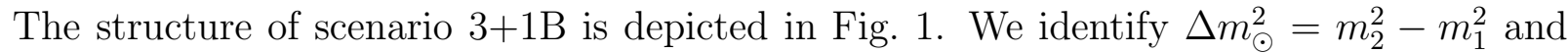
$\Delta m_{\mathrm{A}}^{2}=m_{3}^{2}-m_{2}^{2}$. The heaviest neutrino $m_{4}$ is separated by the LSND scale from the remaining three, which enjoy a normal ordering. We can express $m_{2}, m_{3}$ and $m_{4}$ in terms of the lowest mass $m_{1}$ and the three mass squared differences as

$$
\begin{gathered}
m_{2}=\sqrt{m_{1}^{2}+\Delta m_{\odot}^{2}}, \\
m_{3}=\sqrt{m_{1}^{2}+\Delta m_{\mathrm{A}}^{2}+\Delta m_{\odot}^{2}}, \\
m_{4}=\sqrt{m_{1}^{2}+\Delta m_{\mathrm{LSND}}^{2}} .
\end{gathered}
$$

In Fig. [3] we show - with $\Delta m_{\mathrm{LSND}}^{2}$ taken as $0.9 \mathrm{eV}^{2}$ - the four masses as well as their sum as a function of the smallest mass $m_{1}$. We have "unification" of $m_{2}$ and $m_{1}$ when $m_{1} \gtrsim 0.01$ $\mathrm{eV}$ and of $m_{3}$ and $m_{2}$ when $m_{1} \gtrsim 0.1 \mathrm{eV}$. For small $m_{1} \lesssim 0.01 \mathrm{eV}$ one finds

$$
\Sigma^{3+1 \mathrm{~B}} \simeq \sqrt{\Delta m_{\mathrm{LSND}}^{2}} .
$$

To have $\Sigma \lesssim 1 \mathrm{eV}$, it is required that $m_{1} \lesssim 0.01(0.1) \mathrm{eV}$ if $\Delta m_{\mathrm{LSND}}^{2} \simeq 1(0.2) \mathrm{eV}^{2}$. 
In this scheme it turns out that $\left|U_{e 3}\right|^{2} \simeq \sin ^{2} \theta_{\mathrm{CHOOZ}}$ and $4\left|U_{e 4}\right|^{2}\left|U_{\mu 4}\right|^{2}=\sin ^{2} 2 \theta_{\mathrm{LSND}}$. The remaining elements of the $e$-row of $U$ are $\left|U_{e 1}\right|^{2} \simeq \cos ^{2} \theta_{\odot}$ and $\left|U_{e 2}\right|^{2} \simeq \sin ^{2} \theta_{\odot}$. Neglecting $m_{1}$, we have $m_{2} \simeq \sqrt{\Delta m_{\odot}^{2}}, m_{3} \simeq \sqrt{\Delta m_{\mathrm{A}}^{2}}$ and $m_{4} \simeq \sqrt{\Delta m_{\mathrm{LSND}}^{2}}$. In this case, we can decompose the effective mass in a term well-known from three-flavor analyzes and a contribution from the LSND scale [4], namely:

$$
\begin{gathered}
\left.\langle m\rangle^{3+1 \mathrm{~B}} \simeq\left|\langle m\rangle_{3}^{\mathrm{NH}}+\sqrt{\Delta m_{\mathrm{LSND}}^{2}} e^{i \gamma}\right| U_{e 4}\right|^{2} \mid, \text { where } \\
\langle m\rangle_{3}^{\mathrm{NH}} \equiv m_{1} \cos ^{2} \theta_{\odot}+\sqrt{\Delta m_{\odot}^{2}} e^{i \alpha} \sin ^{2} \theta_{\odot}+\sqrt{\Delta m_{\mathrm{A}}^{2}} e^{i \beta} \sin ^{2} \theta_{\mathrm{CHOOZ}} .
\end{gathered}
$$

The term $\left|\langle m\rangle_{3}^{\mathrm{NH}}\right|$ corresponds to the effective mass in case of three neutrinos with a normal hierarchy. The maximal value of $\left|\langle m\rangle_{3}^{\mathrm{NH}}\right|$ in the range of $m_{1}^{2} \ll \Delta m_{\odot}^{2}$ is known to be less than $0.007 \mathrm{eV}$ [61, 62], whereas $\sqrt{\Delta m_{\mathrm{LSND}}^{2}}\left|U_{e 4}\right|^{2} \simeq 0.008 \mathrm{eV}$ and $0.005 \mathrm{eV}$ for the cases with $\Delta m_{\mathrm{LSND}}^{2}=0.9 \mathrm{eV}^{2}$ and $1.8 \mathrm{eV}^{2}$, respectively. Therefore, if $m_{1}$ is negligible and if $\Delta m_{\mathrm{LSND}}^{2}=0.9 \mathrm{eV}^{2}$, there can be no complete cancellation, and a lower (upper) limit on $\langle m\rangle$ of $0.001(0.015) \mathrm{eV}$ can be expected. If $m_{1}$ is negligible and $\Delta m_{\mathrm{LSND}}^{2}=1.8 \mathrm{eV}^{2}$, however, there can be complete cancellation, and the upper limit of $\langle m\rangle$ is $0.012 \mathrm{eV}$. For both values of $\Delta m_{\mathrm{LSND}}^{2}$ it turns out that also larger values for $m_{1}$ can lead to a vanishing effective mass, defining a "cancellation regime" in Fig. 5. If we vary $\Delta m_{\mathrm{LSND}}^{2}$ in the range $(0.2-0.5) \mathrm{eV}^{2}$ and $m_{1}$ is small, then $\langle m\rangle$ is controlled by the term $\left|U_{e 4}\right|^{2} \sqrt{\Delta m_{\mathrm{LSND}}^{2}}$, which is between 0.02 and $0.07 \mathrm{eV}$, and consequently there is no complete cancellation. For higher values of $m_{1}$ there can be complete cancellation in this case also. If the LSND scale is fixed to 0.9 or $1.8 \mathrm{eV}^{2}$ and $m_{1} \gtrsim 0.1 \mathrm{eV}$, then the masses $m_{1,2,3}$ are quasi-degenerate and their contribution to the effective mass dominates the contribution from $m_{4}$. Consequently, the effective mass in this case is $\langle m\rangle^{3+1 \mathrm{~B}} \simeq m_{0}\left(1-\sin ^{2} 2 \theta_{\odot} \sin ^{2} \alpha / 2\right)$, where $m_{0}$ denotes the common mass scale of the three lightest neutrinos and $\theta_{\mathrm{CHOOZ}}$ has been neglected. For the MiniBooNE range of $(0.2-0.5) \mathrm{eV}^{2}$ this happens for slightly larger values of $m_{1}$, but the important aspect that the effective mass is non-zero in this case holds as well. The reason for this is that solar neutrino mixing is non-maximal. All the discussed features are reflected in Figure 5 .

The kinematic neutrino mass is for $m_{1}=0$

$$
m_{\beta}^{3+1 \mathrm{~B}} \simeq \sqrt{\Delta m_{\odot}^{2} \sin ^{2} \theta_{\odot}+\sin ^{2} \theta_{\mathrm{CHOOZ}} \Delta m_{\mathrm{A}}^{2}+\left|U_{e 4}\right|^{2} \Delta m_{\mathrm{LSND}}^{2}}
$$

which is essentially determined by the term $\sqrt{\Delta m_{\mathrm{LSND}}^{2}}\left|U_{e 4}\right|$. For $\Delta m_{\mathrm{LSND}}^{2}=0.9 \mathrm{eV}^{2}$ as well as $\Delta m_{\mathrm{LSND}}^{2}=1.8 \mathrm{eV}^{2}$ this product is $\simeq 0.085 \mathrm{eV}$, whereas for $\Delta m_{\mathrm{LSND}}^{2}$ in the range $(0.2-0.5) \mathrm{eV}^{2}$ it can vary between $(0.1-0.2) \mathrm{eV}$. This is reproduced in Figure 5 . It is to be noted that $m_{\beta}^{3+1 \mathrm{~B}}$ can be one order of magnitude larger than the maximal effective mass. We can summarize scenario $3+1 \mathrm{~B}$ for small $m_{1}$ as

$$
\Sigma^{3+1 \mathrm{~B}} \simeq \sqrt{\Delta m_{\mathrm{LSND}}^{2}}>m_{\beta}^{3+1 \mathrm{~B}} \gg\langle m\rangle^{3+1 \mathrm{~B}} .
$$

Looking at Figs. 3 and [5, we can make the following statements: if cosmology improves the limit on $\Sigma$ to be below $\sqrt{\Delta m_{\mathrm{LSND}}^{2}}$, scenario $3+1 \mathrm{~B}$ can be ruled out. An effective mass 


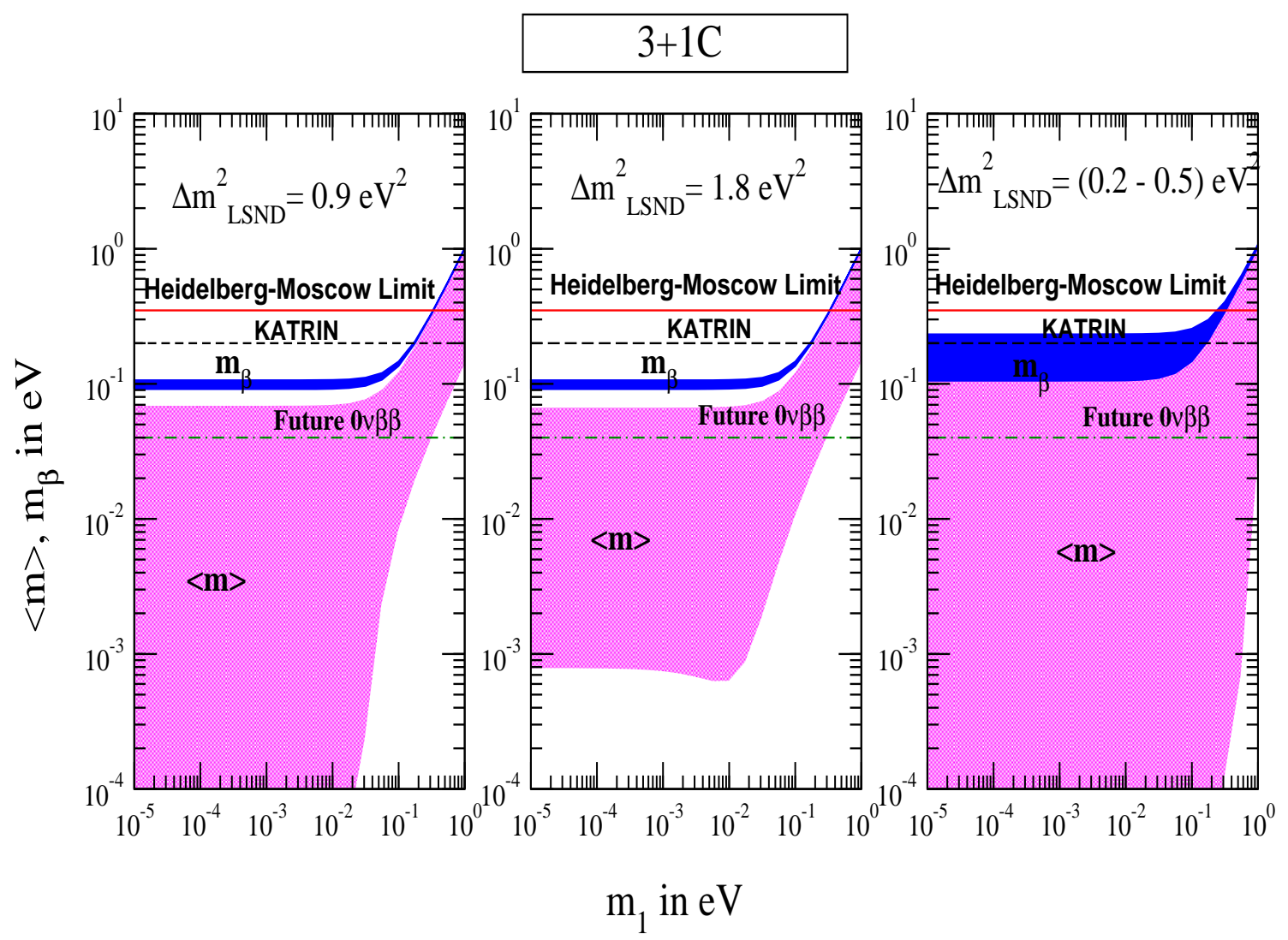

Figure 6: Same as previous Figure for scenario 3+1C.

above $0.05(0.01) \mathrm{eV}$ rules out both overlap points at $\Delta m_{\mathrm{LSND}}^{2}=0.9$ and $1.8 \mathrm{eV}^{2}$ when $m_{1} \lesssim 0.1(0.01) \mathrm{eV}$ is assumed to be small. In this case a successful KATRIN search will make this also possible.

\subsection{Neutrino Masses and Neutrinoless Double Beta Decay in Scenario $3+1 \mathrm{C}$}

The mass spectrum for the scenario $3+1 \mathrm{C}$ is depicted in Fig. [1. We identify $\Delta m_{\odot}^{2}=m_{3}^{2}-m_{2}^{2}$ and $\Delta m_{\mathrm{A}}^{2}=m_{3}^{2}-m_{1}^{2}$. The heaviest neutrino $m_{4}$ is separated by the LSND scale from the remaining three, which enjoy an inverted mass ordering. With this identification the different masses can be expressed in terms of the smallest mass $m_{1}$ and the mass squared differences as

$$
\begin{gathered}
m_{2}=\sqrt{m_{1}^{2}-\Delta m_{\odot}^{2}+\Delta m_{\mathrm{A}}^{2}}, \\
m_{3}=\sqrt{m_{1}^{2}+\Delta m_{\mathrm{A}}^{2}}, \\
m_{4}=\sqrt{m_{1}^{2}+\Delta m_{\mathrm{LSND}}^{2}} .
\end{gathered}
$$

In Fig. [3 we show the four masses as well as their sum as a function of the smallest mass $m_{1}$. We always have quasi-degeneracy between $m_{2}$ and $m_{3}$, and $m_{1}$ is quasi-degenerate 
with them once it is in the vicinity of $0.1 \mathrm{eV}$. The results on the total sum of masses are hardly distinguishable from $3+1 \mathrm{~B}$,

$$
\Sigma^{3+1 \mathrm{C}} \simeq \sqrt{\Delta m_{\mathrm{LSND}}^{2}}
$$

For the mixing matrix elements one finds $\left|U_{e 1}\right|^{2} \simeq \sin ^{2} \theta_{\mathrm{CHOOZ}}$ as well as $4\left|U_{e 4}\right|^{2}\left|U_{\mu 4}\right|^{2}=$ $\sin ^{2} 2 \theta_{\mathrm{LSND}}$ and $\left|U_{e 2}\right|^{2} \simeq \cos ^{2} \theta_{\odot}$ and $\left|U_{e 3}\right|^{2} \simeq \sin ^{2} \theta_{\odot}$.

We show in Fig. [6 our result for the effective mass as well as for the kinematic mass as a function of the smallest mass. The effective mass in the limit of small $m_{1}$ is

$$
\left.\langle m\rangle^{3+1 \mathrm{C}} \simeq\left|\sqrt{\Delta m_{\mathrm{A}}^{2}}\left(e^{i \beta} \cos ^{2} \theta_{\odot}+e^{i \alpha} \sin ^{2} \theta_{\odot}\right)+\sqrt{\Delta m_{\mathrm{LSND}}^{2}} e^{i \gamma}\right| U_{e 4}\right|^{2} \mid,
$$

which can also be written as

$$
\left.\langle m\rangle^{3+1 \mathrm{C}} \simeq\left|\langle m\rangle_{3}^{\mathrm{IH}}+\sqrt{\Delta m_{\mathrm{LSND}}^{2}} e^{i \gamma}\right| U_{e 4}\right|^{2} \mid,
$$

where the term $\left|\langle m\rangle_{3}^{\mathrm{IH}}\right|$ corresponds to the effective mass in case of three neutrinos with an inverted hierarchy [4]. The contribution of the smallest mass $m_{1}$ plays a sub-leading role as it is also multiplied by small $\sin ^{2} \theta_{\mathrm{CHOOZ}}$. The absolute value of $\langle m\rangle_{3}^{\mathrm{IH}}$ is known to be $\sqrt{\Delta m_{\mathrm{A}}^{2}} \sqrt{1-\sin ^{2} 2 \theta_{\odot} \sin ^{2}(\beta-\alpha) / 2}$ and lies (for $m_{1}^{2} \ll \Delta m_{\mathrm{A}}^{2}$ ) between 0.007 and $0.05 \mathrm{eV}$ 61, 62. Whether there is complete cancellation in $\langle m\rangle^{3+1 \mathrm{C}}$ or not depends on the value of $\sqrt{\Delta m_{\mathrm{LSND}}^{2}}\left|U_{e 4}\right|^{2}$. For $\Delta m_{\mathrm{LSND}}^{2}=0.9(1.8) \mathrm{eV}^{2}$ it is given by $\simeq 0.008(0.005) \mathrm{eV}$. Therefore, for $\Delta m_{\mathrm{LSND}}^{2}=0.9 \mathrm{eV}^{2}$ there can be complete cancellation as is seen in the first panel of Fig. 6] but since for $\Delta m_{\mathrm{LSND}}^{2}=1.8 \mathrm{eV}^{2}$ the value of $\sqrt{\Delta m_{\mathrm{LSND}}^{2}}\left|U_{e 4}\right|^{2}$ is less than $0.007 \mathrm{eV}$, there is no complete cancellation in this case (note that it is the other way around in scenario $3+1 \mathrm{~B})$. The lower limit on the effective mass is roughly $10^{-3} \mathrm{eV}$. Similarly, in the case where we vary $\Delta m_{\mathrm{LSND}}^{2}$ from $(0.2-0.5) \mathrm{eV}^{2}$ and $\left|U_{e 4}\right|^{2}$ from 0.05 to 0.1 , the value of the product $\sqrt{\Delta m_{\mathrm{LSND}}^{2}}\left|U_{e 4}\right|^{2}$ can be smaller than $0.06 \mathrm{eV}$ and therefore in this case also there can be complete cancellation over a wide range of $m_{1}$. The maximum value of the effective mass in the small $m_{1}$ limit is roughly

$$
\langle m\rangle_{\max }^{3+1 \mathrm{C}} \simeq \sqrt{\Delta m_{\mathrm{A}}^{2}}+\sqrt{\Delta m_{\mathrm{LSND}}^{2}}\left|U_{e 4}\right|^{2}
$$

Since the product $\sqrt{\Delta m_{\mathrm{LSND}}^{2}}\left|U_{e 4}\right|^{2}$ is approximately the same for 0.9 and $1.8 \mathrm{eV}^{2}$, it follows that $\langle m\rangle_{\max }^{3+1 \mathrm{C}}$ takes the same value $(\simeq 0.07 \mathrm{eV})$ in these two cases. If $m_{1}$ is around $0.1 \mathrm{eV}$, then the three lightest masses are quasi-degenerate, and similar comments as for scenario $3+1 \mathrm{~B}$ discussed in the previous Subsection apply.

The kinematic neutrino mass for $m_{1} \simeq 0$ is

$$
m_{\beta}^{3+1 \mathrm{C}} \simeq \sqrt{\Delta m_{\mathrm{A}}^{2}+\left|U_{e 4}\right|^{2} \Delta m_{\mathrm{LSND}}^{2}}
$$

which is somewhat larger than the maximum effective mass. The blue (dark) band in Figure [6] shows $m_{\beta}$ against $m_{1}$. It is to be noted that in the first two columns of Figure 6 
the width of $m_{\beta}$ is due to the variation over the allowed range of $\Delta m_{\mathrm{A}}^{2}$ as $\Delta m_{\mathrm{LSND}}^{2}$ is held fixed in these plots. In the third column the width is due to variation of both $\Delta m_{\mathrm{LSND}}^{2}$ and $\Delta m_{\mathrm{A}}^{2}$. Let us summarize the situation:

$$
\Sigma^{3+1 \mathrm{C}} \simeq \sqrt{\Delta m_{\mathrm{LSND}}^{2}} \gg m_{\beta}^{3+1 \mathrm{C}} \gtrsim\langle m\rangle_{\max }^{3+1 \mathrm{C}}
$$

Ruling out scenario $3+1 \mathrm{C}$ could be achieved if cosmology improves the limit on $\Sigma$ below

$\sqrt{\Delta m_{\mathrm{LSND}}^{2}}$. For small $m_{1} \lesssim 0.1 \mathrm{eV}$ and a successful KATRIN search both overlap points at $\Delta m_{\mathrm{LSND}}^{2}=0.9$ and $1.8 \mathrm{eV}^{2}$ are ruled out. An effective mass above $0.07 \mathrm{eV}$ rules out all three cases under discussion, unless $m_{1} \gtrsim 0.1 \mathrm{eV}$.

\section{Four Neutrino Mass Matrices}

Models incorporating an extra sterile neutrino have been developed in many papers 63 , 64, 65, 66, 67. In this Section we wish to summarize the typical mass matrices that are consistent with the experimental data in $3+1$ scenarios. In particular, we look for simple $U(1)$ flavor symmetries which can force the approximate form of the mass matrices. The mass matrices for the $2+2$ scenarios are discussed in Appendix B.

$3+1$ scenarios have the property that the sterile neutrino does practically not participate in solar and atmospheric neutrino oscillations. Consequently, there is very little dependence on the respective sterile neutrino fraction. Another general aspect of the results in $3+1$ scenarios is that the well-known three-flavor mass and mixing matrices (see the overviews in 68, 62]) are "embedded" in the four-flavor mass and mixing matrices. This means, in particular, that the corrections to the usual three-flavor mass matrix are of order ${ }^{5}$ $m_{4} \sin ^{2} \theta_{\mathrm{LSND}} \sim \lambda^{2} \sqrt{\Delta m_{\mathrm{LSND}}^{2}} \simeq \sqrt{\Delta m_{\odot}^{2}}$. We introduced here a small parameter $\lambda \simeq 0.1$, to estimate the different mass and mixing scales in the four neutrino framework. Both the LSND and the CHOOZ mixing angle are assumed to be of order $\lambda$, and the mass scales are related through $\Delta m_{\odot}^{2} \simeq \lambda^{2} \Delta m_{\mathrm{A}}^{2} \simeq \lambda^{4} \Delta m_{\mathrm{LSND}}^{2}$. In the approximation we are using, terms of order $\lambda^{2} \sqrt{\Delta m_{\mathrm{LSND}}^{2}}$ are subleading.

\subsection{The Mass Matrix in Scenarios 3+1Aa and 3+1Ab}

In case of scheme $3+1 \mathrm{Aa}$, one has

$$
U^{3+1 \mathrm{Aa}} \simeq\left(\begin{array}{cccc}
\lambda & \lambda & \cos \theta_{\odot} & \sin \theta_{\odot} \\
\lambda & \sin \theta_{A} & -\sin \theta_{\odot} \cos \theta_{A} & \cos \theta_{\odot} \cos \theta_{A} \\
\lambda & \cos \theta_{A} & \sin \theta_{\odot} \sin \theta_{A} & -\cos \theta_{\odot} \sin \theta_{A} \\
1 & \lambda & \lambda & \lambda
\end{array}\right) P
$$

where we set the Dirac phases to zero and included small terms of order $\lambda \sim 0.1$ without writing possible order one coefficients. These terms indicate the typical order of both the

\footnotetext{
${ }^{5}$ We have been made aware of an upcoming analysis on this subject 69 .
} 
CHOOZ angle $\sin \theta_{\mathrm{CHOOZ}}$ and the LSND parameter $\sin \theta_{\mathrm{LSND}}$. The above mixing matrix is unitary only to order $\lambda$. In principle, the order one entries receive additional terms of order $\lambda$ to cure this. The following analysis, however, is not harmed by this. With a given mass hierarchy we can obtain now the approximate form of the mass matrix. By looking at Fig. 3. we can see that typically $m_{4} \simeq m_{3} \simeq m_{2} \gg m_{1}$ holds. So, setting $m_{1}=0$, we have

$$
m_{\nu}^{3+1 \mathrm{Aa}} \sim \sqrt{\Delta m_{\mathrm{LSND}}^{2}}\left(\begin{array}{cccc}
e^{i \beta} c_{\odot}^{2}+e^{i \gamma} s_{\odot}^{2} & c_{\mathrm{A}} c_{\odot} s_{\odot}\left(e^{i \beta}-e^{i \gamma}\right) & s_{\mathrm{A}} c_{\odot} s_{\odot}\left(e^{i \beta}-e^{i \gamma}\right) & \lambda \\
\cdot & e^{i \alpha} s_{\mathrm{A}}^{2}+c_{\mathrm{A}}^{2}\left(e^{i \beta} s_{\odot}^{2}+e^{i \gamma} c_{\odot}^{2}\right) & c_{\mathrm{A}} s_{\mathrm{A}}\left(e^{i \alpha}-e^{i \gamma} c_{\odot}^{2}-e^{i \beta} s_{\odot}^{2}\right) & \lambda \\
\cdot & \cdot & e^{i \alpha} c_{\mathrm{A}}^{2}+s_{\mathrm{A}}^{2}\left(e^{i \beta} s_{\odot}^{2}+e^{i \gamma} c_{\odot}^{2}\right) & \lambda \\
\cdot & \cdot & \cdot & 0
\end{array}\right)
$$

Here we have defined $c_{\mathrm{A}}=\cos \theta_{A}$ and $s_{\mathrm{A}}=\sin \theta_{A}$. A matrix with the entries of the $s$ column zero and the remaining elements of order one conserves the flavor charge $L_{s}$.

Obviously, the upper left $3 \times 3$-block of $m_{\nu}$ corresponds to the well-known three flavor mass matrix in case of quasi-degenerate neutrinos. Apart from the usual $\mu-\tau$ exchange symmetry [70, we can have several interesting special cases: depending on the relative $C P$ parities of the three heavy neutrinos, and setting for simplicity $\theta_{\mathrm{A}}=\pi / 4$ and $\theta_{\odot}=\pi / 4$, we can have $3 \times 3$ matrices proportional to the unit matrix $(\alpha=\beta=\gamma=0)$ or with only a non-vanishing ee and $\mu \tau$ element $(\beta=\gamma=\pi$ and $\alpha=0)$ [68].

The mixing matrix in scenario $3+1 \mathrm{Ab}$ is obtained by exchanging the second and fourth row of the mixing matrix, i.e.,

$$
U^{3+1 \mathrm{Ab}} \simeq\left(\begin{array}{cccc}
\lambda & \sin \theta_{\odot} & \cos \theta_{\odot} & \lambda \\
\lambda & \cos \theta_{\odot} \cos \theta_{A} & -\sin \theta_{\odot} \cos \theta_{A} & \sin \theta_{A} \\
\lambda & -\cos \theta_{\odot} \sin \theta_{A} & \sin \theta_{\odot} \sin \theta_{A} & \cos \theta_{A} \\
1 & \lambda & \lambda & \lambda
\end{array}\right) P .
$$

The mass matrix looks identical to case $3+1 \mathrm{Aa}$, the only change being the replacement $\alpha \leftrightarrow \gamma$. This is analogous to the three-flavor case, in which the structure of the mass matrix for quasi-degenerate neutrinos does not depend on whether the neutrinos are normally and inversely ordered.

\subsection{The Mass Matrix in Scenario 3+1B}

The mixing matrix is given by

$$
U^{3+1 \mathrm{~B}} \simeq\left(\begin{array}{cccc}
\cos \theta_{\odot} & \sin \theta_{\odot} & \lambda & \lambda \\
-\sin \theta_{\odot} \cos \theta_{A} & \cos \theta_{\odot} \cos \theta_{A} & \sin \theta_{A} & \lambda \\
\sin \theta_{\odot} \sin \theta_{A} & -\cos \theta_{\odot} \sin \theta_{A} & \cos \theta_{A} & \lambda \\
\lambda & \lambda & \lambda & 1
\end{array}\right) P .
$$

Regarding the mass states, we have for a smallest mass $m_{1} \lesssim 0.005 \mathrm{eV}$ that $m_{4} \simeq$ $\sqrt{\Delta m_{\mathrm{LSND}}^{2}} \gg m_{3} \simeq \sqrt{\Delta m_{\mathrm{A}}^{2}} \simeq m_{4} \lambda \gg m_{2} \simeq \sqrt{\Delta m_{\mathrm{A}}^{2}} \simeq m_{4} \lambda^{2}$. The mass matrix 
then reads

$$
m_{\nu}^{3+1 \mathrm{~B}} \sim \sqrt{\Delta m_{\mathrm{LSND}}^{2}}\left(\begin{array}{cccc}
0 & 0 & 0 & \lambda \\
\cdot & s_{\mathrm{A}}^{2} \lambda & c_{\mathrm{A}} s_{\mathrm{A}} \lambda & \lambda \\
\cdot & \cdot & c_{\mathrm{A}}^{2} \lambda & \lambda \\
\cdot & \cdot & \cdot & 1
\end{array}\right)
$$

Again, the upper left $3 \times 3$-block corresponds to the well-known three-flavor mass matrix, which can be obtained by demanding $L_{e}$ to be conserved ${ }^{6}$. A four-flavor mass matrix with only the $s s$ element non-zero conserves ${ }^{7} L_{e}+L_{\mu}+L_{\tau}$. As long as $m_{1}$ (and therefore also $m_{2}$ and $m_{3}$ ) are one order of magnitude below $m_{4}$, the main structure of the mass matrix remains, i.e., the $s s$ entry is one order of magnitude larger than the remaining ones.

\subsection{The Mass Matrix in Scenario 3+1C}

The mixing matrix is given by

$$
U^{3+1 \mathrm{C}} \simeq\left(\begin{array}{cccc}
\lambda & \cos \theta_{\odot} & \sin \theta_{\odot} & \lambda \\
\sin \theta_{A} & -\sin \theta_{\odot} \cos \theta_{A} & \cos \theta_{\odot} \cos \theta_{A} & \lambda \\
\cos \theta_{A} & \sin \theta_{\odot} \sin \theta_{A} & -\cos \theta_{\odot} \sin \theta_{A} & \lambda \\
\lambda & \lambda & \lambda & 1
\end{array}\right) P
$$

The three light neutrinos correspond approximately to the well-known inverted hierarchy case of three neutrinos. With $m_{1} \simeq 0$ and $m_{3} \simeq m_{2} \simeq \sqrt{\Delta m_{\mathrm{A}}^{2}} \simeq m_{4} \lambda \simeq \sqrt{\Delta m_{\mathrm{LSND}}^{2}} \lambda$, we get

$m_{\nu}^{3+1 \mathrm{C}} \sim \sqrt{\Delta m_{\mathrm{LSND}}^{2}}\left(\begin{array}{cccc}e^{i \alpha} c_{\odot}^{2}+e^{i \beta} s_{\odot}^{2} \lambda & \left(e^{i \alpha}-e^{i \beta}\right) c_{\mathrm{A}} s_{\odot} c_{\odot} \lambda & \left(e^{i \alpha}-e^{i \beta}\right) s_{\mathrm{A}} s_{\odot} c_{\odot} \lambda & \lambda \\ . & \left(e^{i \alpha} s_{\odot}^{2}+e^{i \beta} c_{\odot}^{2}\right) c_{\mathrm{A}}^{2} \lambda & \left(e^{i \alpha} s_{\odot}^{2}+e^{i \beta} c_{\odot}^{2}\right) c_{\mathrm{A}} s_{\mathrm{A}} \lambda & \lambda \\ . & \cdot & \left(e^{i \alpha} s_{\odot}^{2}+e^{i \beta} c_{\odot}^{2}\right) s_{\mathrm{A}}^{2} \lambda & \lambda \\ . & . & . & 1\end{array}\right)$.

The full four-flavor matrix conserves approximately (i.e., when we neglect $\lambda$ ) the flavor charge $L_{e}+L_{\mu}+L_{\tau}$. We have again for the upper left $3 \times 3$ block the well-known three flavor mass matrix of an inverted hierarchy, which displays for $\theta_{\mathrm{A}}=\pi / 4$ a $\mu-\tau$ symmetry. If we set $\theta_{\odot}=\pi / 4$ and choose $^{8} \alpha=0$ and $\beta=\pi$, then all entries except the $s s, e \mu$ and $e \tau$

\footnotetext{
${ }^{6}$ Conservation of $L_{e}$ in case of four flavors would be achieved when the $\mu s$ and $\tau s$ entries are also of order one.

${ }^{7}$ Obviously, conserving $L_{e}+L_{\mu}+L_{\tau}$ in case of three flavors leads to Dirac neutrinos. For four flavor scenarios one would have to ask for $L_{e}+L_{\mu}+L_{\tau}+L_{s}$ conservation.

${ }^{8}$ Note that such a Pseudo-Dirac structure will lead to enhanced stability with respect to radiative corrections.
} 
elements vanish to order $\lambda$ :

$$
m_{\nu}^{3+1 \mathrm{C}} \sim \sqrt{\Delta m_{\mathrm{LSND}}^{2}}\left(\begin{array}{cccc}
0 & 1 & 1 & 0 \\
\cdot & 0 & 0 & 0 \\
\cdot & \cdot & 0 & 0 \\
\cdot & \cdot & \cdot & 1
\end{array}\right) .
$$

The global symmetry forcing this form of the mass matrix is $L_{e}-L_{\mu}-L_{\tau}$, which was introduced first for the three-flavor case [71, but was used also for the four flavor case 67].

\section{Comments on $3+2$ Scenarios}

By means of introducing more sterile neutrinos, the goodness of fit for explaining the LSND and other short-baseline data can, not really surprisingly, be improved. In this respect, the $3+2$ scenario has been put forward to make the interpretation of all neutrino data less problematic [16]. These schemes have three neutrinos actively oscillating among themselves and two additional sterile neutrinos responsible for the LSND anomaly. Models to accommodate $3+2$ scenarios can be found in 72 . With 5 neutrinos participating in neutrino oscillations, 4 independent $\Delta m^{2}$ are present. In addition to the three discussed previously, we have to deal in addition with $\Delta m_{51}^{2}$.

In the analysis of Ref. [16] two best-fit points are given, one of which corresponds to $\Delta m_{51}^{2}=22 \mathrm{eV}^{2}$, which is clearly not consistent with cosmological constraints. The second best-fit value is at

$$
\begin{gathered}
\Delta m_{41}^{2}=0.46 \mathrm{eV}^{2}, \quad \Delta m_{51}^{2}=0.89 \mathrm{eV}^{2} \\
U_{e 4}=0.090, \quad U_{e 5}=0.125, \quad U_{\mu 4}=0.226, \quad U_{\mu 5}=0.160 .
\end{gathered}
$$

This identification of the mixing matrix elements assumes that the three active neutrinos are lighter than the two sterile ones, i.e., a situation resembling scenarios $3+1 \mathrm{~B}$ and $3+1 \mathrm{C}$. We can decompose the effective mass as a term from the three active neutrinos and a term from the two sterile ones, i.e.,

$$
\langle m\rangle^{3+2}=\left|\langle m\rangle^{3 \mathrm{ac}}+\langle m\rangle^{2 \mathrm{st}}\right|
$$

In this case, the two additional mass scales imply an additional contribution to the effective mass, reading

$\langle m\rangle^{2 \mathrm{st}} \equiv\left|U_{e 4}\right|^{2} m_{4} e^{i \gamma}+\left|U_{e 5}\right|^{2} m_{5} e^{i \rho}=\left|U_{e 4}\right|^{2} \sqrt{m_{1}^{2}+\Delta m_{41}^{2}} e^{i \gamma}+\left|U_{e 5}\right|^{2} \sqrt{m_{1}^{2}+\Delta m_{51}^{2}} e^{i \rho}$,

where we have introduced a fourth relevant Majorana phase $\rho$. If $m_{1}=0$, then the best-fit values given above yield $\left|\langle m\rangle^{2 \text { st }}\right| \simeq(0.01-0.02) \mathrm{eV}$, where the range is caused by the effect of the Majorana phases. 
The cosmological mass parameter is $\Sigma \simeq \sqrt{m_{1}^{2}+\Delta m_{41}^{2}}+\sqrt{m_{1}^{2}+\Delta m_{51}^{2}}$, which for $m_{1}=0$ is $1.6 \mathrm{eV}$, remarkably close to the current relevant limit from cosmology $(1.64 \mathrm{eV})$ obtained in Ref. [53].

The contribution to the neutrino mass measurable in KATRIN is roughly

$$
m_{\beta} \simeq \sqrt{\left|U_{e 4}\right|^{2}\left(m_{1}^{2}+\Delta m_{41}^{2}\right)+\left|U_{e 5}\right|^{2}\left(m_{1}^{2}+\Delta m_{51}^{2}\right)}
$$

which for $m_{1}=0$ is $0.1 \mathrm{eV}$, but can reach testable values if $m_{1} \gtrsim 0.1 \mathrm{eV}$.

If the three active neutrinos display an inverted hierarchy, then their contribution $\left|\langle m\rangle^{3 \text { ac }}\right|$ to the effective mass (see scenario $3+1 \mathrm{C}$ in Section 3.3) lies between $\langle m\rangle=\sqrt{\Delta m_{\mathrm{A}}^{2}}$ and $\langle m\rangle=\sqrt{\Delta m_{\mathrm{A}}^{2}} \cos 2 \theta_{\odot}$, or numerically: $(0.007-0.06) \mathrm{eV}$. Complete cancellation between $\langle m\rangle^{3 \mathrm{ac}}$ and $\langle m\rangle^{2 \mathrm{st}}$ is possible. On the other hand, positive interference of the two terms can lead to $\langle m\rangle \simeq 0.08 \mathrm{eV}$. If the three active neutrinos enjoy a normal mass ordering with $m_{1}=0$, then their contribution $\langle m\rangle^{3 \text { ac }}$ to $\langle m\rangle$ does not exceed $0.007 \mathrm{eV}$ (see scenario $3+1 \mathrm{~B}$ in Section 3.2), so that $\langle m\rangle^{2 \text { st }}$ dominates and represents basically the prediction for $\langle m\rangle$. Note however that these considerations take use of the best-fit values of the fourth and fifth neutrino sector. Varying these parameters within their allowed range and/or effects of non-zero $m_{1}$ might easily allow for severe cancellation. Moreover, one might also exchange $\Delta m_{41}^{2}$ and $\Delta m_{51}^{2}$, leading to identical values for $\Sigma$, slightly larger values of $m_{\beta}$ and $\left|\langle m\rangle^{2 \mathrm{st}}\right| \simeq(0.003-0.02) \mathrm{eV}$ (all if $\left.m_{1}=0\right)$. Consequently, the effective mass vanishes for both orderings of the three active neutrinos. The upper limit is $0.02(0.08) \mathrm{eV}$ for normally (inversely) ordered active neutrinos.

Up to now the two sterile neutrinos were assumed be heavier than the active ones. Also possible is that the two additional neutrinos are lighter than the three active ones, thereby resembling scenarios $3+1 \mathrm{Aa}$ and $3+1 \mathrm{Ab}$. For the mixing matrix elements one has to exchange the indices $4 \leftrightarrow 1$ and $5 \leftrightarrow 2$. The three active ones generate an effective mass larger than roughly $\sqrt{\Delta m_{51}^{2}+m_{1}^{2}} \cos 2 \theta_{\odot}$, which for $m_{1}=0$ is $0.1 \mathrm{eV}$ (see Section 3.1). The contribution of the additional neutrinos is significantly suppressed. The KATRIN parameter is approximately $\sqrt{\Delta m_{51}^{2}} \simeq 0.9 \mathrm{eV}$, surely a testable value. Cosmology will have to probe $\Sigma \simeq 3 \sqrt{\Delta m_{51}^{2}} \simeq 2.8 \mathrm{eV}$, which is incompatible already with current limits.

Finally, it should be clear that the known three-flavor mass and mixing matrices are embedded in the $5 \times 5$ mass and mixing matrices. We note that scenarios with 3 or more sterile neutrinos will also show this "embedding".

\section{Conclusions and Summary}

We have examined the constraints on LSND induced scenarios with extra sterile neutrinos from current and future bounds on neutrinoless double beta decay, tritium beta decays and cosmological limits on the sum of neutrino masses. Since $2+2$ scenarios are already disfavored by the present solar and atmospheric data we considered the $3+1$ scenario in the main part of the paper. The values of $\Delta m_{\mathrm{LSND}}^{2}$ considered in our analysis are $0.9 \mathrm{eV}^{2}$ and $1.8 \mathrm{eV}^{2}$, allowed by a combined analysis of SBL + atmospheric and K2K data [14, 15]. 
We also considered $\Delta m_{\mathrm{LSND}}^{2}$ to vary in the range $(0.2-0.5) \mathrm{eV}^{2}$, motivated by MiniBooNE sensitivity plots. For sake of completeness we discuss the $2+2$ scenarios in Appendix B, Within the $3+1$ scenario there are three possibilities, $3+1 \mathrm{Aa}, \mathrm{b}, 3+1 \mathrm{~B}$ and $3+1 \mathrm{C}$. The sum of neutrino masses is $\simeq 3(1,1) \sqrt{\Delta m_{\mathrm{LSND}}^{2}}$, for scenario $3+1 \mathrm{Aa}$, b $(3+1 \mathrm{~B}, 3+1 \mathrm{C})$ neglecting the solar and atmospheric mass differences. The effective mass can be written as a known three-flavor contribution plus an additional term stemming from the LSND scale. Let us summarize the different aspects:

- in the $3+1 \mathrm{Aa}$ and $3+1 \mathrm{Ab}$ scenarios we have three quasi-degenerate neutrinos of mass $\sqrt{\Delta m_{\text {LSND }}^{2}}$ and a fourth state separated from them by the LSND mass scale. The sum of the masses is $\simeq 3 \sqrt{\Delta m_{\text {LSND }}^{2}}$ in the limit where the mass of the lowest state is vanishingly small. This implies small values of $\Delta m_{\mathrm{LSND}}^{2}\left(\simeq 0.1 \mathrm{eV}^{2}\right)$ to comply with the cosmological bound of order $1 \mathrm{eV}$ for the sum total of the masses. For the considered values of $\Delta m_{\mathrm{LSND}}^{2}$ the sum of masses already exceeds the cosmological limit of $\simeq 1 \mathrm{eV}$. Therefore this scenario is highly constrained from cosmology in particular for higher values of $\Delta m_{\mathrm{LSND}}^{2}$. The effective mass measured in neutrinoless double beta decay varies from $\sqrt{\Delta m_{\mathrm{LSND}}^{2}} \cos 2 \theta_{\odot}$ to $\sqrt{\Delta m_{\mathrm{LSND}}^{2}}$. Since $\theta_{\odot}=\pi / 4$ is no longer allowed by the current data, the minimum value of the effective mass is non-zero in this case ${ }^{9}$. Depending on the value of $\Delta m_{\mathrm{LSND}}^{2}$, the lower value of $\langle m\rangle$ varies from $\simeq(0.04-0.2) \mathrm{eV}$. A part of this region is already disfavored by the limit from the Heidelberg-Moscow experiment. Low values of $\sin ^{2} \theta_{\odot}$ and large values of $\Delta m_{\text {LSND }}^{2}$ jeopardize scenarios $3+1 \mathrm{Aa}$ and $3+1 \mathrm{Ab}$ because the minimal $\langle m\rangle$ becomes too large. The neutrino mass measured in beta decay in this scenario is $\simeq \sqrt{\Delta m_{\mathrm{LSND}}^{2}}$ and coincides with the upper limit of the effective mass in neutrinoless double beta decay;

- the $3+1 \mathrm{~B}$ scenario corresponds to three neutrino states with a normal hierarchy separated from a fourth state by the LSND mass scale. For small $m_{1}<0.01 \mathrm{eV}$ the sum of the masses is given by $\Sigma^{3+1 \mathrm{~B}} \simeq \sqrt{\Delta m_{\mathrm{LSND}}^{2}}$. Hence, the cases $\Delta m_{\mathrm{LSND}}^{2}=0.9$ $\mathrm{eV}^{2}$ and $\Delta m_{\mathrm{LSND}}^{2}$ in the range $(0.2-0.5) \mathrm{eV}^{2}$ are consistent with the cosmological mass bound. Whether there is complete cancellation for the effective mass depends upon the product $\left|U_{e 4}\right|^{2} \sqrt{\Delta m_{\mathrm{LSND}}^{2}}$. For our choice of parameters and hierarchical masses we got very low values of $\langle m\rangle$ only if $\Delta m_{\mathrm{LSND}}^{2}=1.8 \mathrm{eV}^{2}$. The mass measured in tritium beta decay is larger than $\langle m\rangle$ for $m_{1} \lesssim 0.1 \mathrm{eV}$;

- scenario $3+1 \mathrm{C}$ corresponds to the usual three generation inverted hierarchy picture plus an additional neutrino at a higher scale separated by the LSND gap. The second and the third state are quasi-degenerate at $\sqrt{\Delta m_{\mathrm{A}}^{2}}$. For $m_{1}>0.1 \mathrm{eV}$ they become quasi-degenerate with the first state. The cosmological constraint on the $3+1 \mathrm{C}$ scenario is approximately the same as for $3+1 \mathrm{~B}$. The prediction for $\langle m\rangle$ can be split into a contribution from a three neutrino inverted hierarchy scenario plus a term $e^{i \gamma}\left|U_{e 4}\right|^{2} \sqrt{\Delta m_{\mathrm{LSND}}^{2}}$. The contribution of the three-flavor term to $\langle m\rangle$ is

\footnotetext{
${ }^{9}$ This also is the case in scenario $2+2 \mathrm{~A}$.
} 
between $(0.007-0.05) \mathrm{eV}$. Hence, in this case we get very low values of $\langle m\rangle$ for $\Delta m_{\mathrm{LSND}}^{2}=0.9 \mathrm{eV}^{2}$ and in the range $(0.2-0.5) \mathrm{eV}^{2}$.

Regarding the four neutrino mass and mixing matrices, $3+1$ scenarios "embed" in their mass and mixing matrices the well-known three-flavor matrices. Corrections to these threeflavor matrices are of order $\sqrt{\Delta m_{\mathrm{LSND}}^{2}} \sin ^{2} \theta_{\mathrm{LSND}}$, i.e., of order $\Delta m_{\odot}^{2}$, for the mass matrix and of order $\sin \theta_{\mathrm{LSND}}$ for the mixing matrix. The $3+1 \mathrm{~B}$ and $3+1 \mathrm{C}$ scenarios can in principle be motivated by an approximate $L_{e}+L_{\mu}+L_{\tau}$ global symmetry ${ }^{10}$, whereas scenarios $3+1$ Aa,b correspond to a $L_{s}$ global symmetry. Scenario $3+1 \mathrm{C}$ can also be motivated by conservation of $L_{e}-L_{\mu}-L_{\tau}$.

\section{Acknowledgments}

The work of S.G. was supported by the Alexander-von-Humboldt-Foundation. The work of W.R. was supported by the "Deutsche Forschungsgemeinschaft" in the "Sonderforschungsbereich 375 für Astroteilchenphysik" and under project number RO-2516/3-1.

Note added: The recent results from three year WMAP data in combination with largescale structure and supernova data give a bound on the sum of three neutrino masses to be $\Sigma m_{\nu}<0.68 \mathrm{eV}$ (95\% C.L.) which is not very different from the first year limit [74. Therefore the bound on the sum of neutrino masses that we use in this paper is not expected to change significantly with this new data. More stringent bounds can be obtained by including the Lyman- $\alpha$ forest data [75].

\section{A Oscillation Probabilities for Short Baseline Expe- riments}

Here we list the relevant probabilities for short-baseline experiments in the $2+2$ and $3+1$ scenarios. The most general expression for neutrino survival or conversion probability for $N$ neutrino generations is given by

$$
P_{\nu_{\alpha} \nu_{\beta}}=\delta_{\alpha \beta}-4 \sum_{j>i} U_{\alpha i} U_{\beta i} U_{\alpha j} U_{\beta j} \sin ^{2}\left(\frac{\pi L}{\lambda_{i j}}\right)
$$

where $i, j$ varies from 1 to $N$ for $N$ generations, and $\lambda_{i j}=2.47\left(E_{\nu} / \mathrm{MeV}\right)\left(\mathrm{eV}^{2} / \Delta m_{i j}^{2}\right)$ m. If the $\Delta m_{i j}^{2}$ corresponds to $\Delta m_{\odot}^{2}, \Delta m_{\mathrm{A}}^{2}$ or $\Delta m_{\mathrm{LSND}}^{2}$, we denote it with $\lambda_{\odot}, \lambda_{\mathrm{A}}$ and $\lambda_{\mathrm{LSND}}$, respectively. The actual form of the various survival and transition probabilities

\footnotetext{
${ }^{10}$ The $2+2$ scenarios, discussed in the Appendix, have other candidates for global symmetries, for instance $L_{e}+L_{\mu}-L_{\tau}-L_{s}$. Moreover, there can be a $\tau$-s exchange symmetry in analogy to the successful $\mu-\tau$ exchange symmetry for three neutrinos.
} 
will depend on the spectrum of $\Delta m^{2}$ chosen. The above Eq. (45) is assuming the $C P$ phases to be zero. Below we list the relevant probabilities for short-baseline experiments in the $2+2$ and $3+1$ scenarios for the reactor experiments Bugey 9], CHOOZ [59] and the accelerator experiments CDHSW [10, LSND [1] and MiniBooNE [54]. The energy and length scales involved are such that one mass scale dominance approximation holds true in most of the following cases (excepting CHOOZ):

- Scenario 3+1Aa:

$$
\begin{gathered}
P_{\bar{\nu}_{e} \bar{\nu}_{e}}=1-4 U_{e 1}^{2}\left(1-U_{e 1}^{2}\right) \sin ^{2}\left(\pi L / \lambda_{\mathrm{LSND}}\right) \quad \text { (Bugey) } \\
P_{\bar{\nu}_{e} \bar{\nu}_{e}}=1-2 U_{e 1}^{2}\left(1-U_{e 1}^{2}\right)-4 U_{e 2}^{2} \sin ^{2}\left(\pi L / \lambda_{\mathrm{A}}\right) \quad(\mathrm{CHOOZ}) \\
P_{\bar{\nu}_{\mu} \bar{\nu}_{\mu}}=1-4 U_{\mu 1}^{2}\left(1-U_{\mu 1}^{2}\right) \sin ^{2}\left(\pi L / \lambda_{\mathrm{LSND}}\right) \quad(\mathrm{CDHSW}) \\
P_{\bar{\nu}_{\mu} \bar{\nu}_{e}}=4 U_{e 1}^{2} U_{\mu 1}^{2} \sin ^{2}\left(\pi L / \lambda_{\mathrm{LSND}}\right) \quad(\mathrm{LSND}, \mathrm{MiniBooNE})
\end{gathered}
$$

The relevant formulae for scenario $3+1 \mathrm{Ab}$ are obtained from the above by replacing $2 \leftrightarrow 4$.

- Scenario 3+1B:

$$
\begin{gathered}
P_{\bar{\nu}_{e} \bar{\nu}_{e}}=1-4 U_{e 4}^{2}\left(1-U_{e 4}^{2}\right) \sin ^{2}\left(\pi L / \lambda_{\mathrm{LSND}}\right) \quad \text { (Bugey) } \\
P_{\bar{\nu}_{e} \bar{\nu}_{e}}=1-2 U_{e 4}^{2}\left(1-U_{e 4}^{2}\right)-4 U_{e 3}^{2} \sin ^{2}\left(\pi L / \lambda_{\mathrm{A}}\right) \quad(\mathrm{CHOOZ}) \\
P_{\bar{\nu}_{\mu} \bar{\nu}_{\mu}}=1-4 U_{\mu 4}^{2}\left(1-U_{\mu 4}^{2}\right) \sin ^{2}\left(\pi L / \lambda_{S B L}\right) \quad(\mathrm{CDHSW}) \\
P_{\bar{\nu}_{\mu} \bar{\nu}_{e}}=4 U_{e 4}^{2} U_{\mu 4}^{2} \sin ^{2}\left(\pi L / \lambda_{\mathrm{LSND}}\right) \quad(\mathrm{LSND}, \mathrm{MiniBooNE})
\end{gathered}
$$

Note that the probabilities in the $3+1 \mathrm{~B}$ picture can be obtained from $3+1$ Aa by replacing $U_{e 1}$ by $U_{e 4}, U_{\mu 1}$ by $U_{\mu 4}$ and $U_{e 2}$ by $U_{e 3}$ in the CHOOZ probability. The probabilities for the scenario $3+1 \mathrm{C}$ are the same as those of $3+1 \mathrm{~B}$ excepting $U_{e 3}^{2}$ in CHOOZ probability is to be replaced by $U_{e 1}^{2}$. In the presence of $C P$ violation the mixing matrix elements are complex. In the above $3+1$ oscillation probabilities one then has to replace $U_{\alpha i}^{2}$ with $\left|U_{\alpha i}\right|^{2}$.

Let us for the sake of completeness also give the oscillation probabilities in the $2+2$ schemes:

- Scenario $2+2 \mathrm{~A}$ :

$$
P_{\bar{\nu}_{e} \bar{\nu}_{e}}=1-4\left(U_{e 1}^{2}+U_{e 2}^{2}\right)\left(1-U_{e 1}^{2}-U_{e 2}^{2}\right) \sin ^{2}\left(\pi L / \lambda_{\mathrm{LSND}}\right)
$$




$$
\begin{gathered}
P_{\bar{\nu}_{e} \bar{\nu}_{e}}=1-2\left(U_{e 1}^{2}+U_{e 2}^{2}\right)\left(1-U_{e 1}^{2}-U_{e 2}^{2}\right)-4 U_{e 1}^{2} U_{e 2}^{2} \sin ^{2}\left(\pi L / \lambda_{\mathrm{A}}\right) \quad(\mathrm{CHOOZ}) \\
P_{\bar{\nu}_{e} \bar{\nu}_{e}}=1-4\left(U_{\mu 1}^{2}+U_{\mu 2}^{2}\right)\left(1-U_{\mu 1}^{2}-U_{\mu 2}^{2}\right) \sin ^{2}\left(\pi L / \lambda_{\mathrm{LSND}}\right) \quad(\mathrm{CDHSW}) \\
P_{\bar{\nu}_{\mu} \bar{\nu}_{e}}=4\left(U_{e 1} U_{\mu 1}+U_{e 2} U_{\mu 2}\right)^{2} \sin ^{2}\left(\pi L / \lambda_{\mathrm{LSND}}\right) \quad(\mathrm{LSND}, \mathrm{MiniBooNE})
\end{gathered}
$$

The survival and oscillation probabilities in scenario $2+2 \mathrm{~B}$ are obtained from those of scenario $2+2 \mathrm{~A}$ by making the change $1 \leftrightarrow 3$ and $2 \leftrightarrow 4$. In case there are Dirac phases in the PMNS matrix, again $U_{\alpha i}^{2}$ will have to be replaced with $\left|U_{\alpha i}\right|^{2}$. In what regards the (LSND, MiniBooNE) probability, the relevant term would read $\left|U_{e 1}^{*} U_{\mu 1}+U_{e 2}^{*} U_{\mu 2}\right|$ instead of $\left(U_{e 1} U_{\mu 1}+U_{e 2} U_{\mu 2}\right)$. 

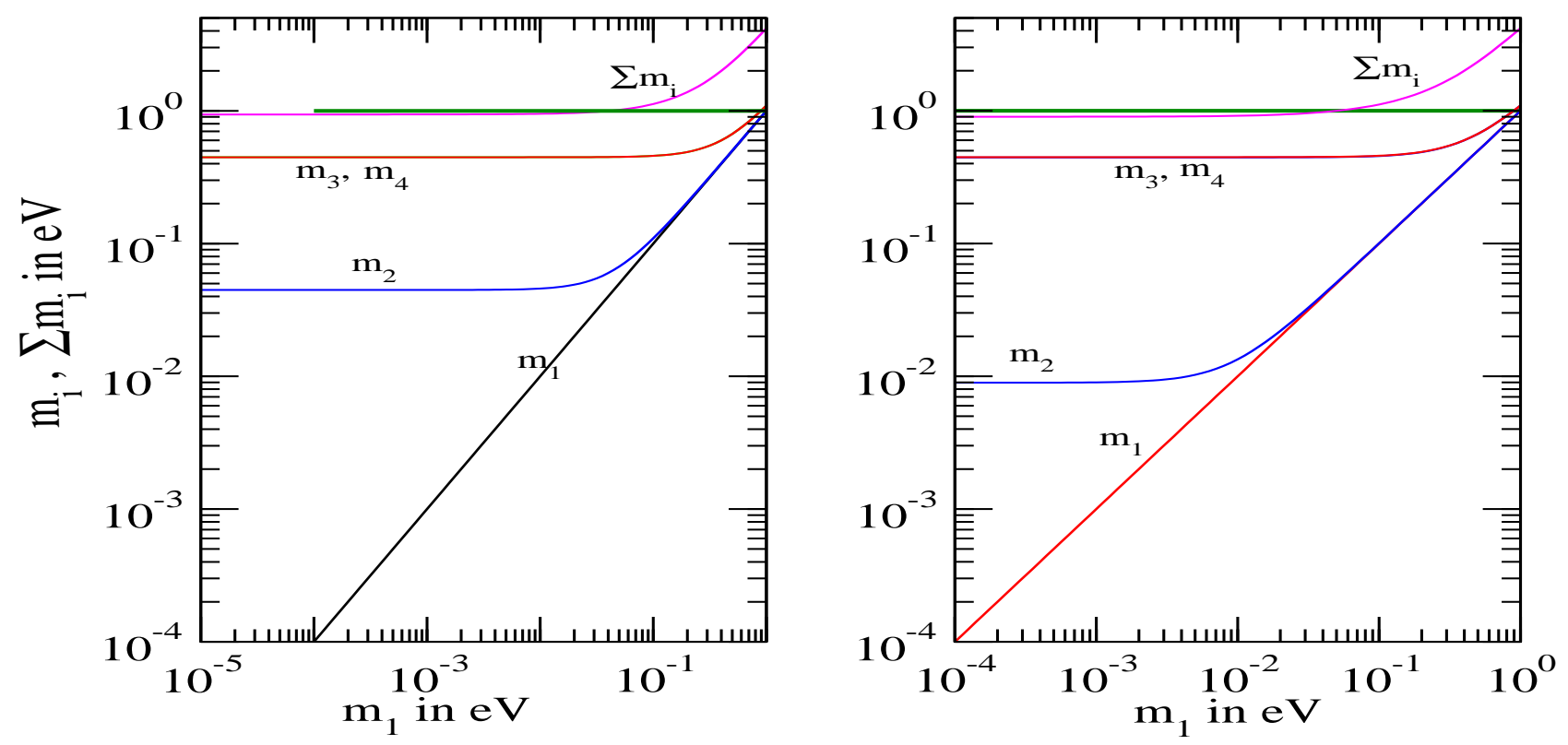

Figure 7: All four neutrino masses and their sum $\Sigma$ against the smallest neutrino mass $m_{1}$ for scenario $2+2 \mathrm{~A}$ (left) and $2+2 \mathrm{~B}$ (right). The masses $m_{3}$ and $m_{4}$ are not distinguishable in the plot. We choose $\Delta m_{\mathrm{LSND}}^{2}=0.2 \mathrm{eV}^{2}$.

\section{B Neutrino Masses and Neutrinoless Double Beta Decay in $2+2$ Scenarios}

The $2+2$ scenarios are disfavored by the combination of solar and atmospheric data regardless if LSND results are confirmed by MiniBooNE or not. However, for the sake of completeness we discuss in this Section the constraints on $2+2$ mass spectra from cosmology, neutrinoless double beta decay and tritium beta decay.

\section{B.1 Neutrino Masses and Neutrinoless Double Beta Decay in Scenario $2+2 \mathrm{~A}$}

On the left side of Fig. 2 the mass ordering of scheme $2+2 \mathrm{~A}$ can be seen. In this scheme it holds that $\Delta m_{\odot}^{2}=\Delta m_{43}^{2}$ and $\Delta m_{\mathrm{A}}^{2}=\Delta m_{21}^{2}$. One has

$$
\begin{gathered}
m_{2}=\sqrt{m_{1}^{2}+\Delta m_{\mathrm{A}}^{2}} \\
m_{3}=\sqrt{m_{1}^{2}+\Delta m_{\mathrm{LSND}}^{2}-\Delta m_{\odot}^{2}}, \\
m_{4}=\sqrt{m_{1}^{2}+\Delta m_{\mathrm{LSND}}^{2}} .
\end{gathered}
$$

The left side of Fig. 7 shows the four mass values and their sum $\Sigma$ as a function of the smallest mass $m_{1}$. Due to $\Delta m_{\mathrm{LSND}}^{2} \gg \Delta m_{\mathrm{A}}^{2}$ the masses $m_{4}$ and $m_{3}$ are hardly distinguishable in the plot and for $m_{1} \gtrsim 0.1 \mathrm{eV}$ the two lightest masses $m_{1}$ and $m_{2}$ become quasi-degenerate. To clarify the role of the cosmology bounds on $\Sigma$, we included in the Figure a value of $\Sigma=1 \mathrm{eV}$. To let the sum of neutrino masses lie below this limit, small values of $\Delta m_{\mathrm{LSND}}^{2}$ and of $m_{1}$ are implied: for small $m_{1} \lesssim 0.05 \mathrm{eV}$ it holds that

$$
m_{4} \simeq m_{3} \simeq \sqrt{\Delta m_{\mathrm{LSND}}^{2}} \gg m_{2,1}
$$




\section{$2+2 \mathrm{~A}$}
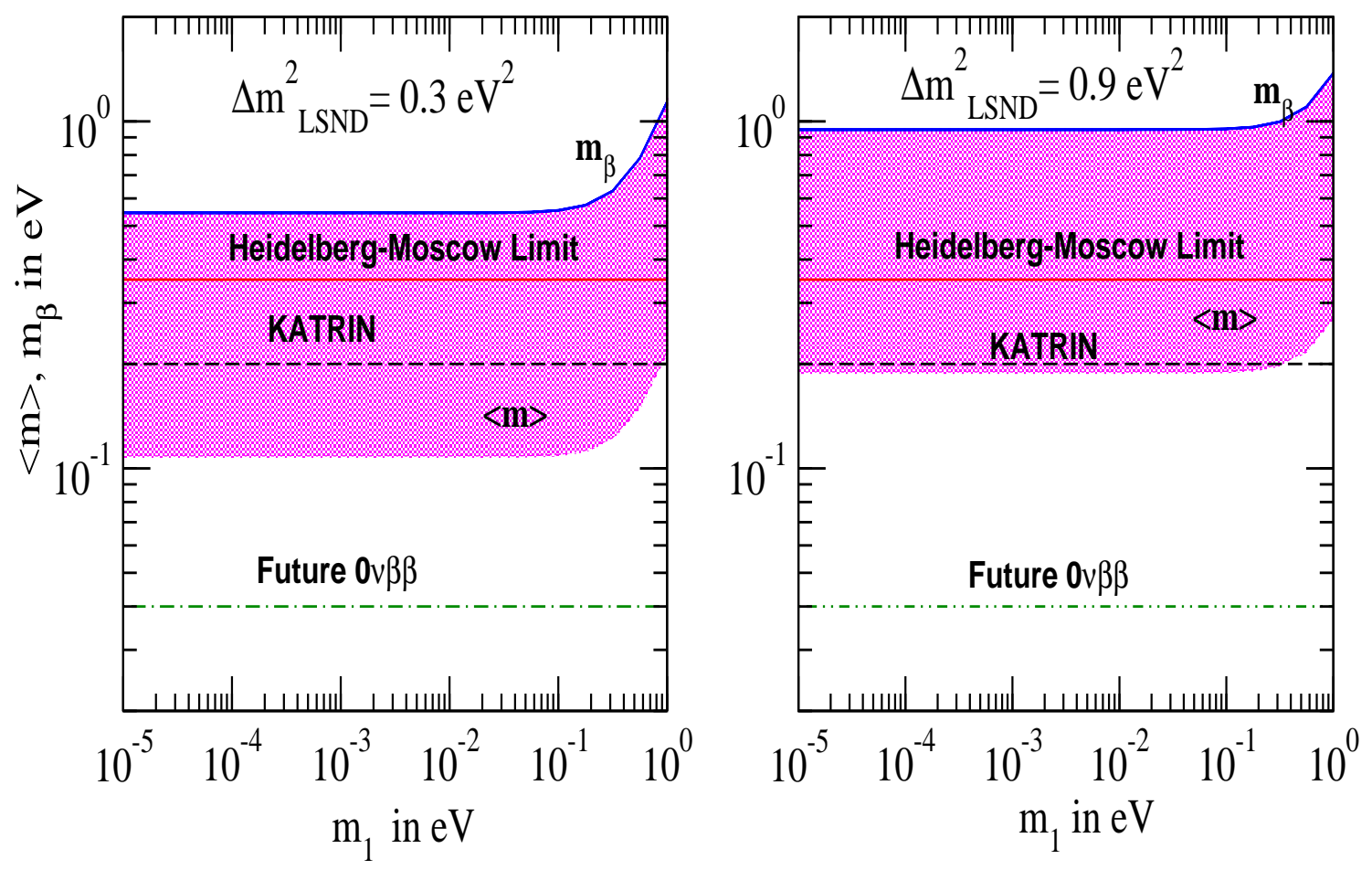

Figure 8: The effective mass $\langle m\rangle$ and the mass $m_{\beta}$ measured in beta decay experiments plotted as a function of the lowest mass in $2+2 \mathrm{~A}$ scenario. The left hand column is for $\Delta m_{\mathrm{LSND}}^{2}=0.3 \mathrm{eV}^{2}$ and $U_{e 1}^{2}=U_{e 2}^{2}=0.002$. The right hand column is for $\Delta m_{\mathrm{LSND}}^{2}=0.9$ $\mathrm{eV}^{2}$ and $U_{e 1}^{2}=U_{e 2}^{2}=0.005$. The other parameters are varied in their current $3 \sigma$ allowed range and all the phases are varied between 0 and $2 \pi$. Also shown is the mass $m_{\beta}$ that will be measured in beta decay experiments, the current and a prospective future limit on the effective mass and the future KATRIN limit.

Hence, $\Sigma \simeq 2 \sqrt{\Delta m_{\mathrm{LSND}}^{2}}$ and therefore low values of $\Delta m_{\mathrm{LSND}}^{2} \lesssim 0.3 \mathrm{eV}^{2}$ are implied by the condition $\Sigma \lesssim 1 \mathrm{eV}$.

Turning to the constraints on the mixing matrix elements, $\left|U_{e 1}\right|^{2}$ and $\left|U_{e 2}\right|^{2}$ are constrained by the short baseline reactor experiment Bugey and the reactor experiment CHOOZ. $\sin ^{2} 2 \theta_{\text {LSND }}$ is given by the combination $\left(U_{e 1} U_{\mu 1}+U_{e 2} U_{\mu 2}\right)^{2}$ and the combination $\left(\left|U_{\mu 1}\right|^{2}+\right.$ $\left.\left|U_{\mu 2}\right|^{2}\right)$ will be constrained by the CDHS experiment. We give the relevant expressions for the probability in Appendix A. Since the one mass scale dominance approximation holds, one can use two-parameter plots to find the constraints on these mixing parameters. However, for the sake of illustration in this paper we use the values of $\Delta m_{\mathrm{LSND}}^{2}$ and $\sin ^{2} 2 \theta_{\mathrm{LSND}}$ from the MiniBooNE sensitivity plot given, e.g., in [73]. We take the representative val- 
ues ${ }^{11}$ for $\left(\Delta m_{\text {LSND }}^{2}, \sin ^{2} 2 \theta_{\text {LSND }}\right)$ as $(0.3,0.02)$ and $(0.9,0.008)$. To extract $U_{e 1}$ and $U_{e 2}$ from $\sin ^{2} 2 \theta_{\text {LSND }}$ we make the plausible assumption $U_{e 1} \simeq U_{e 2}$ and $U_{\mu 1}^{2}=U_{\mu 2}^{2}=0.5$ as implied by atmospheric data. This assumption was for instance used in [43]. With this assumption we have $U_{e 1}^{2}=U_{e 2}^{2}=0.002(0.005)$ for $\Delta m_{\mathrm{LSND}}^{2}=0.9(0.3) \mathrm{eV}^{2}$. In any case it is to be noted that in the $2+2 \mathrm{~A}$ scenario $U_{e 1}$ and $U_{e 2}$ multiply the smaller masses $m_{1}$ and $m_{2}$, and as we will see below their contribution to effective mass as well as the mass measured in beta decay is sub-leading. We furthermore have $\left|U_{e 3}\right| \simeq \cos \theta_{\odot}$ and $\left|U_{e 4}\right| \simeq \sin \theta_{\odot}$.

Since $m_{1}$ and $m_{2}$ are small in scenario $2+2 \mathrm{~A}$ and in addition multiplied with the small elements $\left|U_{e 1}\right|^{2}$ and $\left|U_{e 2}\right|^{2}$, respectively, we can neglect terms including these quantities in what follows. Then the effective mass in scenario $2+2 \mathrm{~A}$ reads

$$
\langle m\rangle^{2+2 \mathrm{~A}} \simeq \sqrt{\Delta m_{\mathrm{LSND}}^{2}} \sqrt{1-\sin ^{2} 2 \theta_{\odot} \sin ^{2}(\beta-\gamma) / 2} .
$$

The non-maximality of solar neutrino mixing implies therefore a non-vanishing effective mass. This is in analogy to the three-flavor case with an inverted hierarchy or quasidegenerate neutrinos. Choosing for instance $\sqrt{\Delta m_{\mathrm{LSND}}^{2}} \simeq 0.5 \mathrm{eV}$ and the values of $\theta_{\odot}$ from Eq. (6), we can predict that

$$
\cos 2 \theta_{\odot} \sqrt{\Delta m_{\mathrm{LSND}}^{2}} \simeq 0.1 \mathrm{eV} \lesssim\langle m\rangle^{2+2 \mathrm{~A}} \lesssim \sqrt{\Delta m_{\mathrm{LSND}}^{2}} \lesssim 0.5 \mathrm{eV} .
$$

This range of $\langle m\rangle$ is well within reach of currently running or planned $0 \nu \beta \beta$ experiments. In Fig. 8] we show the effective mass as a function of the smallest neutrino mass $m_{1}$. We also show the present bound from the Heidelberg-Moscow experiment in this Figure, together with a prospective future limit. It is to be noted that some part of the regions are already disfavored by the Heidelberg-Moscow limit. Thus non-maximality of solar neutrino mixing angle coupled with the existing limit from Heidelberg-Moscow experiment already puts some constraint on the $2+2 \mathrm{~A}$ mass pattern.

Neglecting terms proportional to $U_{e 1}^{2} m_{1}$ and $U_{e 2}^{2} m_{2}$, we have for the kinematic neutrino mass

$$
m_{\beta}^{2+2 \mathrm{~A}} \simeq \sqrt{\left|U_{e 3}\right|^{2} m_{3}^{2}+\left|U_{e 4}\right|^{2} m_{4}^{2}} \simeq \sqrt{\Delta m_{\mathrm{LSND}}^{2}}
$$

Since cosmology implies that $\sqrt{\Delta m_{\text {LSND }}^{2}}$ is below roughly $0.3 \mathrm{eV}$, we expect that $m_{\beta}$ should be close to the lowest value reachable by KATRIN, but close to the current limit on the sum of neutrino masses from cosmology. Since

$$
m_{\beta}^{2+2 \mathrm{~A}} \simeq \sqrt{\Delta m_{\mathrm{LSND}}^{2}} \simeq \Sigma^{2+2 \mathrm{~A}} / 2 \simeq \sqrt{\Delta m_{\mathrm{LSND}}^{2}} \sqrt{1-\sin ^{2} 2 \theta_{\odot} \sin ^{2}(\beta-\gamma) / 2}
$$

one can in principle obtain a set of consistency checks of scenario $2+2 \mathrm{~A}$, and obtain some information on the Majorana phase combination $\beta-\gamma$.

\footnotetext{
${ }^{11}$ It is to be noted that the constraints on mixing angles from SBL experiments are not as severe in the $2+2$ case as in the $3+1$ case.
} 


\section{B.2 Neutrino Masses and Neutrinoless Double Beta Decay in Scenario $2+2 B$}

The right panel of Fig. [ 7 shows the values of the four neutrino masses and their sum $\Sigma$ in scenario $2+2 \mathrm{~B}$, in which one has $\Delta m_{\odot}^{2}=m_{2}^{2}-m_{1}^{2}$ and $\Delta m_{\mathrm{A}}^{2}=m_{4}^{2}-m_{3}^{2}$. We can write the masses of the neutrino states in terms of the smallest mass $m_{1}$ and the three mass squared differences as

$$
\begin{gathered}
m_{2}=\sqrt{m_{1}^{2}+\Delta m_{\odot}^{2}}, \\
m_{3}=\sqrt{m_{1}^{2}+\Delta m_{\mathrm{LSND}}^{2}-\Delta m_{\mathrm{A}}^{2}}, \\
m_{4}=\sqrt{m_{1}^{2}+\Delta m_{\mathrm{LSND}}^{2}} .
\end{gathered}
$$

For the mass values similar statements as for scenario $2+2 \mathrm{~A}$ apply, namely that $m_{4} \simeq m_{3} \simeq$ $\sqrt{\Delta m_{\mathrm{LSND}}^{2}} \simeq \Sigma^{2+2 \mathrm{~B}} / 2$, where $\Delta m_{\mathrm{LSND}}^{2}$ should lie at the lower end of its allowed range in order to obey the constraints from cosmology. The smallest neutrino mass $m_{1}$ should lie below $0.05 \mathrm{eV}$ and therefore $m_{2}$ and $m_{1}$ are typically not very close to each other, unless $m_{1} \simeq 0.02-0.05 \mathrm{eV}$.

In what regards the mixing matrix elements, scenario $2+2 \mathrm{~B}$ is obtained from scenario $2+2 \mathrm{~A}$ by exchanging the indices $1 \leftrightarrow 3$ and $2 \leftrightarrow 4$. Hence, $\left|U_{e 1}\right|$ and $\left|U_{e 2}\right|$ are roughly given by $\cos \theta_{\odot}$ and $\sin \theta_{\odot}$, respectively. The elements $\left|U_{e 3}\right|$ and $\left|U_{e 4}\right|$, however, are implied to be small.

We show in Fig. 9 the effective mass as a function of the smallest mass. The effective mass is approximately given by

$$
\begin{aligned}
& \left.\langle m\rangle^{2+2 \mathrm{~B}} \simeq\left|\cos ^{2} \theta_{\odot} m_{1}+\sin ^{2} \theta_{\odot} m_{2} e^{i \alpha}+\right| U_{e 3}\right|^{2} m_{3} e^{i \beta}+\left|U_{e 4}\right|^{2} m_{4} e^{i \gamma} \mid \\
& \simeq\left|\cos ^{2} \theta_{\odot} m_{1}+\sin ^{2} \theta_{\odot} m_{2} e^{i \alpha}+\sqrt{\Delta m_{\mathrm{LSND}}^{2}}\left(\left|U_{e 3}\right|^{2} e^{i \beta}+\left|U_{e 4}\right|^{2} e^{i \gamma}\right)\right| \\
& \simeq\left|\sin ^{2} \theta_{\odot} \sqrt{\Delta m_{\odot}^{2}}+\sqrt{\Delta m_{\mathrm{LSND}}^{2}}\left(\left|U_{e 3}\right|^{2} e^{i(\beta-\alpha)}+\left|U_{e 4}\right|^{2} e^{i(\gamma-\beta)}\right)\right|
\end{aligned}
$$

where we neglected $m_{1}$ for the last approximation.

Since $U_{e 3}^{2}$ and $U_{e 4}^{2}$ are small, the two large masses $m_{3}$ and $m_{4}$ are multiplied with small mixing matrix elements, whereas the small masses $m_{1}$ and $m_{2}$ are multiplied with large mixing matrix elements. As a consequence, there can be cancellations leading to a very small or zero effective mass. Note the analogy of this situation with the three-flavor case: in the inverted hierarchy the large masses are multiplied with mixing matrix elements corresponding to the large solar neutrino mixing, whose non-maximality allows no cancellation. In the normal hierarchy, the largest mass $m_{3}$ is multiplied with the smallest mixing matrix element, and complete cancellation can occur. Since the degree of cancellation depends on the values of the two small quantities $U_{e 3}^{2}$ and $U_{e 4}^{2}$ for this case, we present our results for $\Delta m_{\mathrm{LSND}}^{2}=0.3 \mathrm{eV}^{2}$ and two different choices for $U_{e 3}^{2}$ and $U_{e 4}^{2}$. In the left panel we show the plot where we assume $U_{e 3}^{2} \simeq U_{e 4}^{2}=0.005$. In this case complete cancellation can occur. In the right panel we present our results with $U_{e 4}^{2}=0$ and $U_{e 3}^{2}=0.01$. In this case the minimal $\langle m\rangle$ has a higher value. The two terms in the last equation have the typical order $\sqrt{\Delta m_{\odot}^{2}} \sin ^{2} \theta_{\odot} \simeq(2-4) \cdot 10^{-3} \mathrm{eV}$ and $\sqrt{\Delta m_{\mathrm{LSND}}^{2}}\left|U_{e 3}\right|^{2} \simeq 0.005 \mathrm{eV}$. The upper limit on 

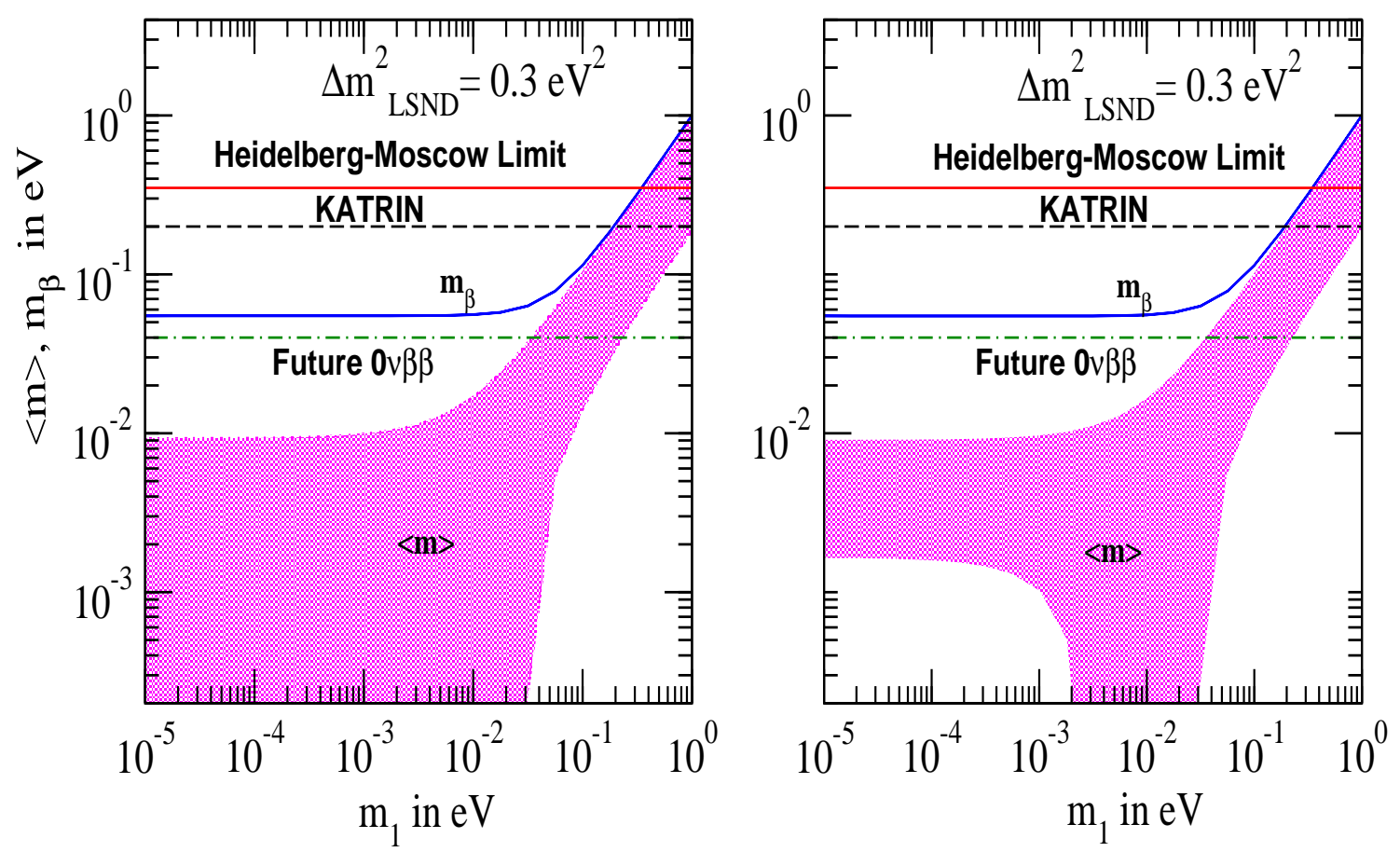

Figure 9: Same as previous Figure for scenario $2+2 \mathrm{~B}$ and $\Delta m_{\mathrm{LSND}}^{2}=0.3 \mathrm{eV}^{2}$. The left hand column is for $U_{e 3}^{2}=U_{e 4}^{2}=0.005$, the right hand column is for $U_{e 4}^{2}=0$ and $U_{e 3}^{2}=0.01$.

$\langle m\rangle^{2+2 \mathrm{~B}}$ is then roughly given by $0.01 \mathrm{eV}$. Hence, we can in principle distinguish scenario $2+2 \mathrm{~A}$ from $2+2 \mathrm{~B}$ via $0 \nu \beta \beta$ as long as $m_{1}$ is small. This is analogous to the situation normal vs. inverted hierarchy in the three flavor case.

The kinematic neutrino mass as measurable in the KATRIN experiment is given by

$$
\begin{aligned}
& m_{\beta}^{2+2 \mathrm{~B}}=\sqrt{\left|U_{e 1}\right|^{2} m_{1}^{2}+\left|U_{e 2}\right|^{2} m_{2}^{2}+\left|U_{e 3}\right|^{2} m_{3}^{2}+\left|U_{e 4}\right|^{2} m_{4}^{2}} \\
& \simeq \sqrt{\cos ^{2} \theta_{\odot} m_{1}^{2}+\sin ^{2} \theta_{\odot} m_{2}^{2}+\Delta m_{\mathrm{LSND}}^{2}\left(\left|U_{e 3}\right|^{2}+\left|U_{e 4}\right|^{2}\right)} \\
& \simeq \sqrt{\Delta m_{\odot}^{2} \sin ^{2} \theta_{\odot}+\Delta m_{\mathrm{LSND}}^{2}\left(\left|U_{e 3}\right|^{2}+\left|U_{e 4}\right|^{2}\right)}
\end{aligned}
$$

where we neglected again $m_{1}$ in the last approximation. Both terms are of similar magnitude and we can expect that $m_{\beta}^{2+2 \mathrm{~B}} \simeq 0.1 \mathrm{eV}$, larger than the effective mass by an order of magnitude and below the future KATRIN limit.

\section{The Mass Matrix in the $2+2$ Scenarios}

Now we discuss the form of the mass matrices in $2+2$ scenarios. Our approach and the approximations made are the same as for the $3+1$ case, and details are given in Section 4 . 


\section{C.1 The Mass Matrix in Scenario 2+2A}

For scenario $2+2 \mathrm{~A}$ we can express the mixing matrix as follows [2, 3]:

$$
U^{2+2 \mathrm{~A}} \simeq\left(\begin{array}{cccc}
\lambda & \lambda & \cos \theta_{\odot} & \sin \theta_{\odot} \\
\cos \theta_{\mathrm{atm}} & \sin \theta_{\mathrm{atm}} & \lambda & \lambda \\
-\sin \eta \sin \theta_{\mathrm{atm}} & \sin \eta \cos \theta_{\mathrm{atm}} & -\cos \eta \sin \theta_{\odot} & \cos \eta \cos \theta_{\odot} \\
-\cos \eta \sin \theta_{\mathrm{atm}} & \cos \eta \cos \theta_{\mathrm{atm}} & \sin \eta \sin \theta_{\odot} & -\sin \eta \cos \theta_{\odot}
\end{array}\right) P .
$$

The parameter $\eta$ indicates inasmuch sterile neutrinos participate in atmospheric or solar neutrino oscillations. For $\eta=0$ atmospheric neutrinos oscillate completely into sterile ones and for $\eta=\pi / 2$ solar neutrinos oscillate into sterile ones. With a given mass hierarchy we can obtain now the approximate form of the mass matrix. Glancing at Fig. [7] we identify two interesting possibilities, namely

$$
\begin{aligned}
& \text { (i) } \sqrt{\Delta m_{\mathrm{LSND}}^{2}} \simeq m_{4} \simeq m_{3} \gg m_{2} \simeq \sqrt{\Delta m_{\odot}^{2}} \gg m_{1}, \\
& \text { (ii) } \sqrt{\Delta m_{\mathrm{LSND}}^{2}} \simeq m_{4} \simeq m_{3} \gg m_{2} \simeq m_{1} \simeq 0.1 \mathrm{eV} .
\end{aligned}
$$

The first case (i) corresponds to a very small mass $m_{1}$ and the second one (ii) to two quasi-degenerate pairs, though only a small range of $m_{1}$ values allows for this possibility. Since the form of $m_{\nu}$ is similar in both cases, we mainly discuss case (i). We have then $m_{2} \simeq m_{4} \lambda^{2}$ and the mass matrix reads

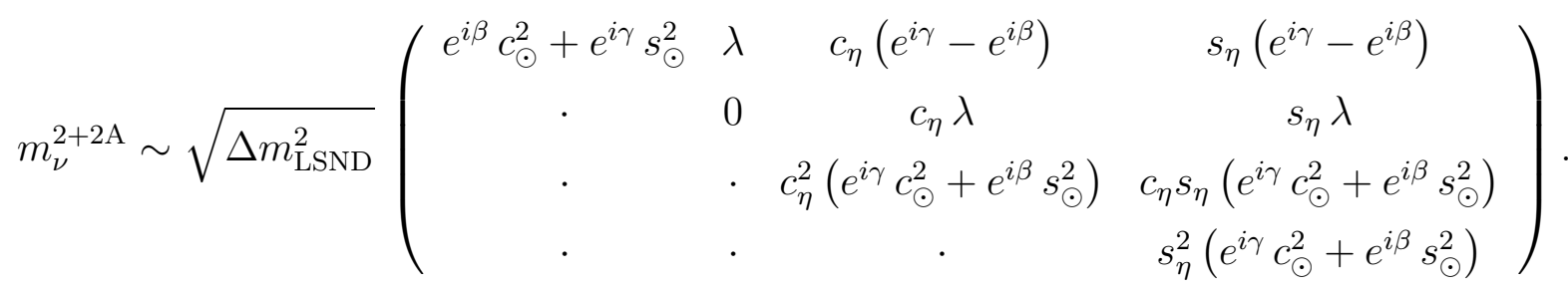

We defined the obvious notation $c_{\odot}=\cos \theta_{\odot}$ and $s_{\odot}=\sin \theta_{\odot}$. Terms of order $\lambda^{2}$ and unimportant factors, such as a coefficient $c_{1} e^{i \gamma} c_{\odot}-c_{2} e^{i \beta} s_{\odot}$ for the $\mu \tau$ element, are not included in our expressions. The factors $c_{1,2}$ depend on the precise values of the CHOOZ and the LSND angles. Note that contributions of the atmospheric mixing are suppressed in the mass matrix. If the heavy states $m_{3}$ and $m_{4}$ have equal $C P$ parities, or when $\beta=\gamma$, then this leads to the vanishing of the $e \tau$ and es entries of $m_{\nu}$, independent of $\eta$. In case of opposite $C P$ parities of $\nu_{3}$ and $\nu_{4}$ (which would imply enhanced stability with respect to radiative corrections), the ee element and the $\tau s$ block of $m_{\nu}$ would be slightly suppressed by a factor $\cos 2 \theta_{\odot}$. Assuming as yet another approximation that $\theta_{\odot}=\pi / 4$ would make these entries vanish. Several special cases can be obtained from the above matrix. For instance, if solar neutrinos oscillate entirely in sterile neutrinos, i.e., $\eta=\pi / 2$, then

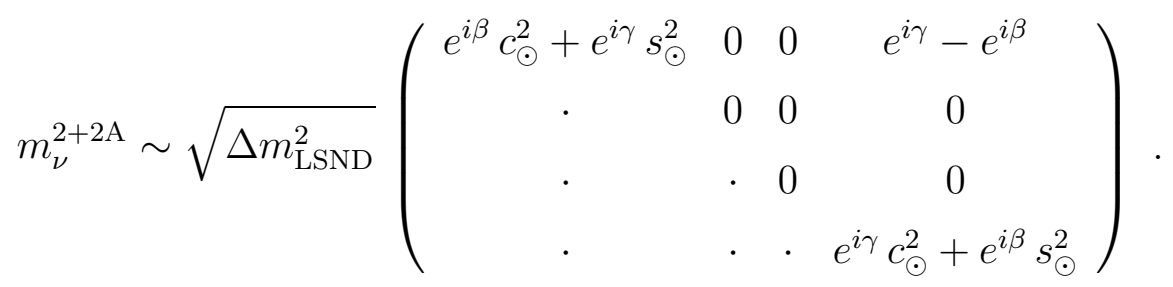


This matrix conserves the flavor charge $L_{\mu}+L_{\tau}$. If we choose equal $C P$ parities of $\nu_{3}$ and $\nu_{4}$, or when $\beta=\gamma$, then the e $\tau$ entry vanishes.

In analogy, if atmospheric neutrinos oscillate entirely in sterile neutrinos $(\eta=0)$ then one finds

$$
m_{\nu}^{2+2 \mathrm{~A}} \sim \sqrt{\Delta m_{\mathrm{LSND}}^{2}}\left(\begin{array}{cccc}
e^{i \beta} c_{\odot}^{2}+e^{i \gamma} s_{\odot}^{2} & 0 & e^{i \gamma}-e^{i \beta} & 0 \\
\cdot & 0 & 0 & 0 \\
\cdot & \cdot & e^{i \gamma} c_{\odot}^{2}+e^{i \beta} s_{\odot}^{2} & 0 \\
\cdot & \cdot & \cdot & 0
\end{array}\right) .
$$

Opposite $C P$ parities (a Pseudo-Dirac structure) of $\nu_{3}$ and $\nu_{4}$ will again lead to $\left(m_{\nu}\right)_{e \tau}=0$. Now consider $c_{\eta} \simeq s_{\eta}$. The mass matrix takes the form

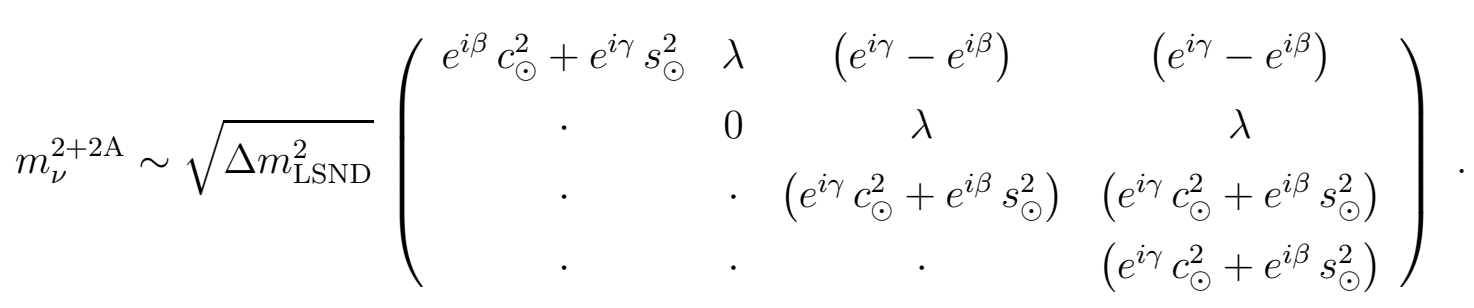

We therefore find an approximate $\tau$-s symmetry, in analogy to the successful $\mu-\tau$ symmetry of the three neutrino case [70]. It is present when sterile neutrinos participate equally in solar and atmospheric neutrino oscillations. The $\tau$-s symmetry does strictly speaking only say that $\left(m_{\nu}\right)_{\tau \tau}=\left(m_{\nu}\right)_{s s}$, here it holds in addition that $\left(m_{\nu}\right)_{\tau s}=\left(m_{\nu}\right)_{s s}$. If we consider the matrix

$$
m_{\nu}=\left(\begin{array}{cccc}
a & b & d & d \\
\cdot & h & e & e \\
\cdot & \cdot & f & f \\
\cdot & \cdot & \cdot & f
\end{array}\right)
$$

we see that one eigenvalue is zero. Setting for simplicity $b=h=e=0$ (these entries are suppressed in the previous equation), leads to two vanishing mass eigenvalues and $m_{3,4}=\frac{1}{2}\left(a+2 f \mp \sqrt{8 d^{2}+(a-2 f)^{2}}\right)$, and therefore $\Delta m_{\odot}^{2}=(a+2 f) \sqrt{8 d^{2}+(a-2 f)^{2}}$. In this limit the atmospheric $\Delta m^{2}$ is vanishing. We have $U_{e 1}=U_{e 2}=0,\left|U_{e 3}\right|^{2}=\frac{1}{2}-$ $(a / 2-f) /\left(\sqrt{8 d^{2}+(a-2 f)^{2}}\right)$ and $\left|U_{\mu 1}\right|=\left|U_{\tau 1}\right|=1 / \sqrt{2}$. Hence, the approximate form of Eq. (56) is almost reproduced when $a \simeq-2 f$. Small breaking terms can in principle help to reach full agreement.

The second interesting case (ii) occurs when $m_{4} \simeq m_{3} \gg m_{2} \simeq m_{1} \simeq \lambda m_{4} \simeq 0.1 \mathrm{eV}$. The implications are similar to case (i), but for completeness we give the resulting form of the 
mass matrix:

$$
m_{\nu}^{2+2 \mathrm{~A}} \sim \sqrt{\Delta m_{\mathrm{LSND}}^{2}}\left(\begin{array}{cccc}
e^{i \beta} c_{\odot}^{2}+e^{i \gamma} s_{\odot}^{2} & \lambda & c_{\eta}\left(e^{i \gamma}-e^{i \beta}\right) & s_{\eta}\left(e^{i \gamma}-e^{i \beta}\right) \\
\cdot & \lambda & s_{\eta} c_{\mathrm{atm}} s_{\mathrm{atm}} & s_{\eta} \\
\cdot & \cdot & c_{\eta}^{2}\left(e^{i \gamma} c_{\odot}^{2}+e^{i \beta} s_{\odot}^{2}\right) & c_{\eta} s_{\eta}\left(e^{i \gamma} c_{\odot}^{2}+e^{i \beta} s_{\odot}^{2}\right) \\
\cdot & \cdot & \cdot & s_{\eta}^{2}\left(e^{i \gamma} c_{\odot}^{2}+e^{i \beta} s_{\odot}^{2}\right)
\end{array}\right) .
$$

Comparing with case (i), we see that the second row of the mass matrix differs. It vanishes to first order when $\eta=0$. If $\eta \neq 0$ the atmospheric neutrino mixing angle has some dependence on the form of the mass matrix. As an additional approximation, let us take $\theta_{\odot}=\pi / 4$. Then, for $e^{i \gamma}+e^{i \beta}=0$, i.e., a Pseudo-Dirac structure of the two heavy masses, and $c_{\eta} \simeq s_{\eta}$, we have

$$
m_{\nu}^{2+2 \mathrm{~A}} \sim \sqrt{\Delta m_{\mathrm{LSND}}^{2}}\left(\begin{array}{cccc}
0 & 0 & 1 & 1 \\
\cdot & 0 & 1 & 1 \\
\cdot & \cdot & 0 & 0 \\
\cdot & \cdot & \cdot & 0
\end{array}\right)
$$

This matrix conserves $L_{e}+L_{\mu}-L_{\tau}-L_{s}$. Indeed, this global symmetry has been used in 64] and, in somewhat different form in [65], to explain the neutrino data including LSND. Moreover, the above matrix has all diagonal entries zero, a property typically shared by radiative models of neutrino mass generation. In Ref. [66] such a case is treated.

\section{C.2 The Mass Matrix in Scenario 2+2B}

For scenario $2+2 \mathrm{~B}$ we can express the mixing matrix by exchanging in the mixing matrix from scheme $2+2 \mathrm{~A}$ the indices $1 \leftrightarrow 3$ and $2 \leftrightarrow 4$. Hence,

$$
U^{2+2 \mathrm{~B}} \simeq\left(\begin{array}{cccc}
\cos \theta_{\odot} & \sin \theta_{\odot} & \lambda & \lambda \\
\lambda & \lambda & \cos \theta_{\mathrm{atm}} & \sin \theta_{\mathrm{atm}} \\
-\cos \eta \sin \theta_{\odot} & \cos \eta \cos \theta_{\odot} & -\sin \eta \sin \theta_{\mathrm{atm}} & \sin \eta \cos \theta_{\mathrm{atm}} \\
\sin \eta \sin \theta_{\odot} & -\sin \eta \cos \theta_{\odot} & -\cos \eta \sin \theta_{\mathrm{atm}} & \cos \eta \cos \theta_{\mathrm{atm}}
\end{array}\right) P .
$$

Here we again put terms of order $\lambda \sim 0.1$, which is the typical order of both the CHOOZ angle and the LSND parameter. We have again two cases of interest:

(i) $\sqrt{\Delta m_{\mathrm{LSND}}^{2}} \simeq m_{4} \simeq m_{3} \gg m_{2} \simeq \sqrt{\Delta m_{\odot}^{2}} \gg m_{1}$,

$$
\text { (ii) } \sqrt{\Delta m_{\mathrm{LSND}}^{2}} \simeq m_{4} \simeq m_{3} \gg m_{2} \simeq m_{1} \simeq 0.1 \mathrm{eV} \text {. }
$$

Let us start with case (i), for which

$$
m_{\nu}^{2+2 \mathrm{~B}} \sim \sqrt{\Delta m_{\mathrm{LSND}}^{2}}\left(\begin{array}{cccc}
0 & \lambda & s_{\eta} \lambda & c_{\eta} \lambda \\
\cdot & c_{\mathrm{atm}}^{2} e^{i \beta}+s_{\mathrm{atm}}^{2} e^{i \gamma} & c_{\mathrm{atm}} s_{\mathrm{atm}} s_{\eta}\left(e^{i \beta}-e^{i \gamma}\right) & c_{\mathrm{atm}} s_{\mathrm{atm}} c_{\eta}\left(e^{i \beta}-e^{i \gamma}\right) \\
\cdot & \cdot & s_{\eta}^{2}\left(c_{\mathrm{atm}}^{2} e^{i \gamma}+s_{\mathrm{atm}}^{2} e^{i \beta}\right) & c_{\eta} s_{\eta}\left(c_{\mathrm{atm}}^{2} e^{i \gamma}+s_{\mathrm{atm}}^{2} e^{i \beta}\right) \\
\cdot & \cdot & \cdot & c_{\eta}^{2}\left(c_{\mathrm{atm}}^{2} e^{i \gamma}+s_{\mathrm{atm}}^{2} e^{i \beta}\right)
\end{array}\right)
$$


In contrast to scenario $2+2 \mathrm{~A}$ it is the solar neutrino mixing angle whose contribution to the mass matrix is suppressed. Note again the approximate $\tau$ - $s$ exchange symmetry of the mass matrix in case of $s_{\eta} \simeq c_{\eta}$. The (close-to-)maximality of $\theta_{\text {atm }}$ allows to further simplify the mass matrix to

$$
m_{\nu}^{2+2 \mathrm{~B}} \sim \sqrt{\Delta m_{\mathrm{LSND}}^{2}}\left(\begin{array}{cccc}
0 & \lambda & s_{\eta} \lambda & c_{\eta} \lambda \\
\cdot & e^{i \beta}+e^{i \gamma} & s_{\eta}\left(e^{i \beta}-e^{i \gamma}\right) & c_{\eta}\left(e^{i \beta}-e^{i \gamma}\right) \\
\cdot & \cdot & s_{\eta}^{2}\left(e^{i \gamma}+e^{i \beta}\right) & c_{\eta} s_{\eta}\left(e^{i \gamma}+e^{i \beta}\right) \\
\cdot & . & . & c_{\eta}^{2}\left(e^{i \gamma}+e^{i \beta}\right)
\end{array}\right) .
$$

Opposite (identical) $C P$ parities of $\nu_{3}$ and $\nu_{4}$ lead to a vanishing $\mu \mu$ entry and $\tau s$ block ( $\mu \tau$ and $\mu s$ elements). If we indeed impose a Pseudo-Dirac structure on $\nu_{3}$ and $\nu_{4}$, then we have

$$
m_{\nu}^{2+2 \mathrm{~B}} \sim \sqrt{\Delta m_{\mathrm{LSND}}^{2}}\left(\begin{array}{cccc}
0 & \lambda & s_{\eta} \lambda & c_{\eta} \lambda \\
\cdot & 0 & s_{\eta} & c_{\eta} \\
\cdot & 0 & 0 \\
\cdot & \cdot & 0
\end{array}\right)
$$

If $c_{\eta} \simeq s_{\eta}$ and the $e \mu$ entry of $m_{\nu}$ (recall that there can be a coefficient) is more suppressed than the $e \tau$ and es elements, then we have again a mass matrix conserving $L_{e}+L_{\mu}-L_{\tau}-L_{s}$. Setting $\eta=0$ (atmospheric-sterile oscillations) and $\theta_{\text {atm }}=\pi / 4$, then at leading order

$$
m_{\nu}^{2+2 \mathrm{~B}} \sim \sqrt{\Delta m_{\mathrm{LSND}}^{2}}\left(\begin{array}{cccc}
0 & 0 & 0 & 0 \\
\cdot & e^{i \beta}+e^{i \gamma} & 0 & e^{i \beta}-e^{i \gamma} \\
\cdot & \cdot & 0 & 0 \\
\cdot & \cdot & \cdot & e^{i \beta}+e^{i \gamma}
\end{array}\right)
$$

conserving $L_{e}+L_{\tau}$. Recall that $\eta=\pi / 2$ in scenario $2+2 \mathrm{~A}$ lead to conservation of $L_{\mu}+L_{\tau}$. By choosing opposite or identical $C P$ parities one can further simplify the mass matrix. If $\eta=\pi / 2$ (solar-sterile oscillations), then at leading order

$$
m_{\nu}^{2+2 \mathrm{~B}} \sim \sqrt{\Delta m_{\mathrm{LSND}}^{2}}\left(\begin{array}{cccc}
0 & 0 & 0 & 0 \\
\cdot & e^{i \beta}+e^{i \gamma} & e^{i \beta}-e^{i \gamma} & 0 \\
\cdot & \cdot & e^{i \beta}+e^{i \gamma} & 0 \\
\cdot & \cdot & \cdot & 0
\end{array}\right)
$$

conserving $L_{e}+L_{s}$.

Finally, we note that case (ii), defined by $m_{4} \simeq m_{3} \gg m_{2} \simeq m_{1} \simeq \lambda m_{4} \simeq 0.1 \mathrm{eV}$, leads to 


$$
\begin{aligned}
& m_{\nu}^{2+2 \mathrm{~B}} \sim \sqrt{\Delta m_{\mathrm{LSND}}^{2}}\left(\begin{array}{cccc}
\lambda & \lambda & s_{\eta} & c_{\eta} \\
\cdot & c_{\mathrm{atm}}^{2} e^{i \beta}+s_{\mathrm{atm}}^{2} e^{i \gamma} & c_{\mathrm{atm}} s_{\mathrm{atm}} s_{\eta}\left(e^{i \beta}-e^{i \gamma}\right) & c_{\mathrm{atm}} s_{\mathrm{atm}} c_{\eta}\left(e^{i \beta}-e^{i \gamma}\right) \\
\cdot & \cdot & s_{\eta}^{2}\left(c_{\mathrm{atm}}^{2} e^{i \gamma}+s_{\mathrm{atm}}^{2} e^{i \beta}\right) & c_{\eta} s_{\eta}\left(c_{\mathrm{atm}}^{2} e^{i \gamma}+s_{\mathrm{atm}}^{2} e^{i \beta}\right) \\
\cdot & \cdot & \cdot & c_{\eta}^{2}\left(c_{\mathrm{atm}}^{2} e^{i \gamma}+s_{\mathrm{atm}}^{2} e^{i \beta}\right)
\end{array}\right) \\
& \simeq\left(\begin{array}{cccc}
\lambda & \lambda & s_{\eta} & c_{\eta} \\
\cdot & e^{i \beta}+e^{i \gamma} & s_{\eta}\left(e^{i \beta}-e^{i \gamma}\right) & c_{\eta}\left(e^{i \beta}-e^{i \gamma}\right) \\
\cdot & \cdot & s_{\eta}^{2}\left(e^{i \gamma}+e^{i \beta}\right) & c_{\eta} s_{\eta}\left(e^{i \gamma}+e^{i \beta}\right) \\
\cdot & \cdot & \cdot & c_{\eta}^{2}\left(e^{i \gamma}+e^{i \beta}\right)
\end{array}\right)
\end{aligned}
$$

where we set $\theta_{\text {atm }}=\pi / 4$. The difference with respect to case (i) lies in the electron row of $m_{\nu}$. Nevertheless, for $s_{\eta} \simeq c_{\eta}$ and $e^{i \beta}+e^{i \gamma}$ one encounters again an approximate $L_{e}+L_{\mu}-L_{\tau}-L_{s}$ symmetry. Setting $\eta=0$ gives

$$
m_{\nu}^{2+2 \mathrm{~B}} \sim \sqrt{\Delta m_{\mathrm{LSND}}^{2}}\left(\begin{array}{cccc}
\lambda & \lambda & 0 & 1 \\
\cdot & e^{i \beta}+e^{i \gamma} & 0 & e^{i \beta}-e^{i \gamma} \\
\cdot & \cdot & 0 & 0 \\
\cdot & \cdot & \cdot & e^{i \beta}+e^{i \gamma}
\end{array}\right)
$$

conserving $L_{\tau}$, if the $e e$ and $e \mu$ entries are not too strongly suppressed. On the other hand, for $\eta=\pi / 2$ one finds

$$
m_{\nu}^{2+2 \mathrm{~B}} \sim \sqrt{\Delta m_{\mathrm{LSND}}^{2}}\left(\begin{array}{cccc}
\lambda & \lambda & 1 & 0 \\
\cdot & e^{i \beta}+e^{i \gamma} & e^{i \beta}-e^{i \gamma} & 0 \\
\cdot & \cdot & e^{i \beta}+e^{i \gamma} & 0 \\
\cdot & \cdot & \cdot & 0
\end{array}\right)
$$

i.e., a matrix conserving $L_{s}$ when the $e e$ and $e \mu$ entries are not too strongly suppressed.

\section{References}

[1] C. Athanassopoulos et al. [LSND Collaboration], Phys. Rev. Lett. 75, 2650 (1995), A. Aguilar et al. [LSND Collaboration], Phys. Rev. D 64, 112007 (2001); C. Athanassopoulos et al. [LSND Collaboration], Phys. Rev. Lett. 81, 1774 (1998); Phys. Rev. C 54, 2685 (1996).

[2] J. J. Gomez-Cadenas and M. C. Gonzalez-Garcia, Z. Phys. C 71, 443 (1996);

S. Goswami, Phys. Rev. D 55, 2931 (1997). 
[3] S. M. Bilenky, C. Giunti and W. Grimus, Eur. Phys. J. C 1, 247 (1998); V. D. Barger et al., Phys. Lett. B 489, 345 (2000); O. L. G. Peres and A. Y. Smirnov, Nucl. Phys. B 599, 3 (2001); W. Grimus and T. Schwetz, Eur. Phys. J. C 20, 1 (2001); C. Giunti and M. Laveder, JHEP 0102, 001 (2001).

[4] E. D. Church et al., Phys. Rev. D 66, 013001 (2002).

[5] Also see C. Giunti, Mod. Phys. Lett. A 18, 1179 (2003).

[6] K. S. Babu, R. K. Schaefer and Q. Shafi, Phys. Rev. D 53, 606 (1996); N. Okada and O. Yasuda, Int. J. Mod. Phys. A 12, 3669 (1997); S. M. Bilenky, C. Giunti, W. Grimus and T. Schwetz, Astropart. Phys. 11, 413 (1999).

[7] More recent analyzes are A. Pierce and H. Murayama, Phys. Lett. B 581, 218 (2004); M. Cirelli, G. Marandella, A. Strumia and F. Vissani, Nucl. Phys. B 708, 215 (2005).

[8] B. Armbruster et al. [KARMEN Collaboration], Phys. Rev. D 65, 112001 (2002).

[9] Y. Declais et al., Nucl. Phys. B 434, 503 (1995).

[10] F. Dydak et al., Phys. Lett. B 134, 281 (1984).

[11] S. Fukuda et al. [Super-Kamiokande Collaboration], Phys. Rev. Lett. 85, 3999 (2000).

[12] Q. R. Ahmad et al. [SNO Collaboration], Phys. Rev. Lett. 87, 071301 (2001); Phys. Rev. Lett. 89, 011301 (2002); S. N. Ahmed et al. [SNO Collaboration], Phys. Rev. Lett. 92, 181301 (2004).

[13] M. C. Gonzalez-Garcia, M. Maltoni and C. Pena-Garay, Phys. Rev. D 64, 093001 (2001).

[14] M. Maltoni, T. Schwetz, M. A. Tortola and J. W. F. Valle, Nucl. Phys. B 643, 321 (2002).

[15] M. Maltoni, T. Schwetz, M. A. Tortola and J. W. F. Valle, New J. Phys. 6, 122 (2004).

[16] M. Sorel, J. M. Conrad and M. Shaevitz, Phys. Rev. D 70, 073004 (2004).

[17] H. Murayama and T. Yanagida, Phys. Lett. B 520, 263 (2001); A. Strumia, Phys. Lett. B 539, 91 (2002); G. Barenboim, L. Borissov and J. Lykken, hep-ph/0212116; M. C. Gonzalez-Garcia, M. Maltoni and T. Schwetz, Phys. Rev. D 68, 053007 (2003); V. Barger, D. Marfatia and K. Whisnant, Phys. Lett. B 576, 303 (2003).

[18] G. Barenboim and N. E. Mavromatos, JHEP 0501, 034 (2005).

[19] D. B. Kaplan, A. E. Nelson and N. Weiner, Phys. Rev. Lett. 93, 091801 (2004).

[20] E. Ma, G. Rajasekaran and I. Stancu, Phys. Rev. D 61, 071302 (2000); E. Ma and G. Rajasekaran, Phys. Rev. D 64, 117303 (2001). 
[21] K. S. Babu and S. Pakvasa, hep-ph/0204236.

[22] S. Palomares-Ruiz, S. Pascoli and T. Schwetz, JHEP 0509, 048 (2005).

[23] H. Päs, S. Pakvasa and T. J. Weiler, Phys. Rev. D 72, 095017 (2005).

[24] C. Kraus et al., Eur. Phys. J. C 40, 447 (2005).

[25] A. Osipowicz et al. [KATRIN Collaboration], hep-ex/0109033; G. Drexlin [KATRIN Collaboration], Nucl. Phys. Proc. Suppl. 145, 263 (2005).

[26] J. Schechter and J. W. F. Valle, Phys. Rev. D 22, 2227 (1980).

[27] H. V. Klapdor-Kleingrothaus et al., Eur. Phys. J. A 12, 147 (2001).

[28] C. E. Aalseth et al. [IGEX Collaboration], Phys. Rev. D 65, 092007 (2002).

[29] Y. Shitov [NEMO Collaboration], nucl-ex/0405030.

[30] S. Capelli, hep-ex/0505045 and hep-ex/0501034.

[31] R. Ardito et al., hep-ex/0501010.

[32] R. Gaitskell et al. [Majorana Collaboration], nucl-ex/0311013.

[33] I. Abt et al., hep-ex/0404039.

[34] M. Danilov et al., Phys. Lett. B 480, 12 (2000).

[35] H. Ejiri et al., Phys. Rev. Lett. 85, 2917 (2000).

[36] K. Zuber, Phys. Lett. B 519, 1 (2001).

[37] N. Ishihara, T. Ohama and Y. Yamada, Nucl. Instrum. Meth. A 373, 325 (1996).

[38] S. Yoshida et al., Nucl. Phys. Proc. Suppl. 138, 214 (2005).

[39] G. Bellini et al., Eur. Phys. J. C 19, 43 (2001).

[40] C. Aalseth et al., hep-ph/0412300.

[41] H. V. Klapdor-Kleingrothaus et al., Mod. Phys. Lett. A 16, 2409 (2001); C. E. Aalseth et al., Mod. Phys. Lett. A 17, 1475 (2002); H. L. Harney, hep-ph/0205293; H. V. Klapdor-Kleingrothaus, hep-ph/0205228, F. Feruglio, A. Strumia and F. Vissani, Nucl. Phys. B 637, 345 (2002), [Addendum-ibid. B 659, 359 (2003)]; H. V. Klapdor-Kleingrothaus et al., Phys. Lett. B 586, 198 (2004). 
[42] C. Giunti, Phys. Rev. D 61, 036002 (2000); S. M. Bilenky, C. Giunti, W. Grimus, B. Kayser and S. T. Petcov, Phys. Lett. B 465, 193 (1999); A. Kalliomaki and J. Maalampi, Phys. Lett. B 484, 64 (2000); Y. Farzan, O. L. G. Peres and A. Y. Smirnov, Nucl. Phys. B 612, 59 (2001); S. Pakvasa and P. Roy, Phys. Lett. B 535, 181 (2002).

[43] H. V. Klapdor-Kleingrothaus, H. Päs and A. Y. Smirnov, Phys. Rev. D 63, 073005 (2001).

[44] S. M. Bilenky, S. Pascoli and S. T. Petcov, Phys. Rev. D 64, 113003 (2001).

[45] V. Barger, J. P. Kneller, H. S. Lee, D. Marfatia and G. Steigman, Phys. Lett. B 566, 8 (2003).

[46] R. H. Cyburt et al., Astropart. Phys. 23, 313 (2005);

[47] S. Hannestad, astro-ph/0511595.

[48] S. Hannestad and G. Raffelt, JCAP 0404, 008 (2004).

[49] S. Dodelson, A. Melchiorri and A. Slosar, astro-ph/0511500.

[50] R. Foot and R. R. Volkas, Phys. Rev. Lett. 75, 4350 (1995).

[51] G. Gelmini, S. Palomares-Ruiz and S. Pascoli, Phys. Rev. Lett. 93, 081302 (2004).

[52] J. F. Beacom, N. F. Bell and S. Dodelson, Phys. Rev. Lett. 93, 121302 (2004); N. F. Bell, E. Pierpaoli and K. Sigurdson, astro-ph/0511410.

[53] S. Hannestad, hep-ph/0602058 and references therein.

[54] M. H. Shaevitz [BooNE Collaboration], Nucl. Phys. Proc. Suppl. 145, 208 (2005).

[55] B. Pontecorvo, Zh. Eksp. Teor. Fiz. 33, 549 (1957) and 34, 247 (1958); Z. Maki, M. Nakagawa and S. Sakata, Prog. Theor. Phys. 28, 870 (1962).

[56] S. M. Bilenky, J. Hosek and S. T. Petcov, Phys. Lett. B 94, 495 (1980); M. Doi et al., Phys. Lett. B 102, 323 (1981); J. Schechter and J. W. F. Valle, Phys. Rev. D 23, 1666 (1981).

[57] S. Goswami, talk given at XXII International Symposium on Lepton-Photon Interactions at High Energy "Lepton/Photon 05", Uppsala, Sweden, July 2005; http://lp2005.tsl.uu.se/ lp2005/LP2005/programme/index.htm; S. Choubey, hep-ph/0509217.

[58] G. L. Fogli, E. Lisi, A. Marrone and A. Palazzo, hep-ph/0506083.

[59] M. Apollonio et al., Eur. Phys. J. C 27, 331 (2003); F. Boehm et al., Phys. Rev. D 64, 112001 (2001). 
[60] F. Vissani, Nucl. Phys. Proc. Suppl. 100, 273 (2001) hep-ph/0012018; Y. Farzan and A. Y. Smirnov, Phys. Lett. B 557, 224 (2003).

[61] S. Pascoli, S. T. Petcov and T. Schwetz, hep-ph/0505226; M. Lindner, A. Merle and W. Rodejohann, Phys. Rev. D 73, 053005 (2006).

[62] S. Choubey and W. Rodejohann, Phys. Rev. D 72, 033016 (2005).

[63] A probably still incomplete list: J. T. Peltoniemi, D. Tommasini and J. W. F. Valle, Phys. Lett. B 298, 383 (1993); J. T. Peltoniemi and J. W. F. Valle, Nucl. Phys. B 406, 409 (1993); R. Foot and R. R. Volkas, Phys. Rev. D 52, 6595 (1995); Z. G. Berezhiani and R. N. Mohapatra, Phys. Rev. D 52, 6607 (1995); E. Ma and P. Roy, Phys. Rev. D 52, 4780 (1995); E. Ma, Phys. Lett. B 380, 286 (1996); E. J. Chun, A. S. Joshipura and A. Y. Smirnov, Phys. Rev. D 54, 4654 (1996); Phys. Lett. B 357, 608 (1995); D. Suematsu, Phys. Lett. B 392, 413 (1997); K. Benakli and A. Y. Smirnov, Phys. Rev. Lett. 79, 4314 (1997); E. J. Chun, C. W. Kim and U. W. Lee, Phys. Rev. D 58, 093003 (1998); N. Arkani-Hamed and Y. Grossman, Phys. Lett. B 459, 179 (1999); S. Mohanty, D. P. Roy and U. Sarkar, Phys. Lett. B 445, 185 (1998); P. Langacker, Phys. Rev. D 58, 093017 (1998) M. Bando and K. Yoshioka, Prog. Theor. Phys. 100, 1239 (1998); G. R. Dvali and Y. Nir, JHEP 9810, 014 (1998); C. Liu and J. H. Song, Phys. Rev. D 60, 036002 (1999); W. Grimus, R. Pfeiffer and T. Schwetz, Eur. Phys. J. C 13, 125 (2000). Z. Chacko and R. N. Mohapatra, Phys. Rev. D 61, 053002 (2000); K. S. Babu and T. Yanagida, Phys. Lett. B 491, 148 (2000); D. O. Caldwell, R. N. Mohapatra and S. J. Yellin, Phys. Rev. Lett. 87, 041601 (2001); F. Borzumati, K. Hamaguchi and T. Yanagida, Phys. Lett. B 497, 259 (2001); A. Perez-Lorenzana and C. A. De S. Pires, Phys. Lett. B 522, 297 (2001); B. Brahmachari, S. Choubey and R. N. Mohapatra, Phys. Lett. B 536, 94 (2002); K. L. McDonald and B. H. J. McKellar, hep-ph/0401073. G. J. . Stephenson, T. Goldman, B. H. J. McKellar and M. Garbutt, Int. J. Mod. Phys. A 20, 6373 (2005); K. S. Babu and G. Seidl, Phys. Rev. D 70, 113014 (2004); M. Frank, I. Turan and M. Sher, Phys. Rev. D 71, 113001 (2005); Phys. Rev. D 71, 113002 (2005) J. Sayre, S. Wiesenfeldt and S. Willenbrock, Phys. Rev. D 72, 015001 (2005); A. de Gouvea, Phys. Rev. D 72, 033005 (2005); J. Kang and T. Li, Phys. Rev. D 71, 095011 (2005); A. G. Dias, C. A. de S.Pires and P. S. Rodrigues da Silva, Phys. Lett. B 628, 85 (2005).

[64] S. Goswami and A. S. Joshipura, Phys. Rev. D 65, 073025 (2002).

[65] K. S. Babu and R. N. Mohapatra, Phys. Lett. B 522, 287 (2001).

[66] N. Gaur, A. Ghosal, E. Ma and P. Roy, Phys. Rev. D 58, 071301 (1998); Y. Okamoto and M. Yasue, Prog. Theor. Phys. 101, 1119 (1999); P. Roy and S. K. Vempati, Phys. Rev. D 65, 073011 (2002).

[67] R. N. Mohapatra, Phys. Rev. D 64, 091301 (2001); R. N. Mohapatra, S. Nasri and H. B. Yu, Phys. Rev. D 72, 033007 (2005). 
[68] R. N. Mohapatra et al., hep-ph/0510213 G. Altarelli and F. Feruglio, Phys. Rept. 320 (1999) 295; New J. Phys. 6, 106 (2004); W. Rodejohann, Phys. Rev. D 69, 033005 (2004); S. F. King, Rept. Prog. Phys. 67, 107 (2004); see also N. Haba, J. Sato, M. Tanimoto and K. Yoshioka, Phys. Rev. D 64, 113016 (2001).

[69] A. Smirnov, talk given at 9th International Conference On Astroparticle And Underground Physics (TAUP 2005), Zaragoza, Spain, 10-14 September 2005.

[70] C. S. Lam, Phys. Lett. B 507, 214 (2001); W. Grimus and L. Lavoura, JHEP 0107, 045 (2001); E. Ma, Phys. Rev. D 66, 117301 (2002); P. F. Harrison and W. G. Scott, Phys. Lett. B 547, 219 (2002); an incomplete list of more recent studies is: Y. Koide, Phys. Rev. D 69, 093001 (2004); W. Grimus et al., Nucl. Phys. B 713, 151 (2005); R. N. Mohapatra, JHEP 0410, 027 (2004); I. Aizawa, T. Kitabayashi and M. Yasue, Phys. Rev. D 72, 055014 (2005); C. S. Lam, Phys. Rev. D 71, 093001 (2005); R. N. Mohapatra and W. Rodejohann, Phys. Rev. D 72, 053001 (2005); I. Aizawa and M. Yasue, Phys. Rev. D 73, 015002 (2006).

[71] S. T. Petcov, Phys. Lett. B 110 (1982) 245; an incomplete list of more recent studies is: G. K. Leontaris, J. Rizos and A. Psallidas, Phys. Lett. B 597, 182 (2004); P. H. Frampton and R. N. Mohapatra, JHEP 0501, 025 (2005); S. T. Petcov and W. Rodejohann, Phys. Rev. D 71, 073002 (2005); W. Grimus and L. Lavoura, J. Phys. G 31, 683 (2005); G. Altarelli and R. Franceschini, hep-ph/0512202.

[72] G. J. Stephenson, T. Goldman, B. H. J. McKellar and M. Garbutt, hep-ph/0307245; K. S. Babu and G. Seidl, Phys. Lett. B 591, 127 (2004); K. L. McDonald, B. H. J. McKellar and A. Mastrano, Phys. Rev. D 70, 053012 (2004); W. Krolikowski, Acta Phys. Polon. B 35, 1675 (2004); R. N. Mohapatra, S. Nasri and H. B. Yu in Ref. 67]; W. Krolikowski, hep-ph/0506099.

[73] M. Sorel, FERMILAB-THESIS-2005-07

[74] D. N. Spergel et al., astro-ph/0603449.

[75] U. Seljak, A. Slosar and P. McDonald, astro-ph/0604335. 